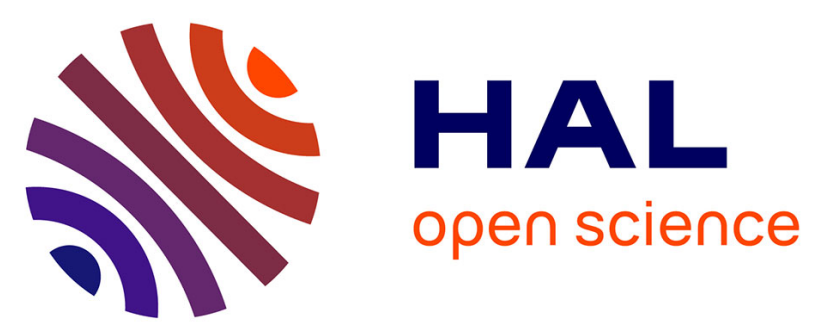

\title{
Measurement of the Layer Compressibility Modulus of a Lamellar Mesophase with a Surface Forces Apparatus
}

P. Richetti, P. Kékicheff, P. Barois

\section{To cite this version:}

P. Richetti, P. Kékicheff, P. Barois. Measurement of the Layer Compressibility Modulus of a Lamellar Mesophase with a Surface Forces Apparatus. Journal de Physique II, 1995, 5 (8), pp.1129-1154. 10.1051/jp2:1995173 . jpa-00248224

\section{HAL Id: jpa-00248224 https://hal.science/jpa-00248224}

Submitted on 1 Jan 1995

HAL is a multi-disciplinary open access archive for the deposit and dissemination of scientific research documents, whether they are published or not. The documents may come from teaching and research institutions in France or abroad, or from public or private research centers.
L'archive ouverte pluridisciplinaire HAL, est destinée au dépôt et à la diffusion de documents scientifiques de niveau recherche, publiés ou non, émanant des établissements d'enseignement et de recherche français ou étrangers, des laboratoires publics ou privés. 
Classification

Physics Abstracts

$61.30-\mathrm{v}-61.30 \mathrm{Jf}-68.15+\mathrm{e}$

\title{
Measurement of the Layer Compressibility Modulus of a Lamellar Mesophase with a Surface Forces Apparatus
}

\author{
P. Richetti $\left({ }^{1}\right)$, P. Kékicheff $\left({ }^{2}\right)$ and P. Barois $\left({ }^{1}\right)$ \\ $\left({ }^{1}\right)$ Centre de Recherche Paul Pascal, Avenue A. Schweitzer, 33600 Pessac, France \\ $\left({ }^{2}\right)$ Department of Applied Mathematics, Australian National University, Research School of \\ Physical Sciences and Engineering, Canberra A.C.T. 0200, Australia
}

(Received 1 December 1994, received in final form 25 April 1995, accepted 2 May 1995)

Résumé. - A l'aide d'un appareil de mesures de force, nous avons contraint une phase lamellaire. Le profil de force mesuré entre les deux surfaces de l'appareil ımmergées dans la mésophase est périodıque, oscillant, amorti et discontinu. Nous avons développé un modèle qui considère la géométrie spécifique des surfaces du montage. Un réseau de boucles de dislocation concentriques est retenu afin de remplir l'espacement d'épaisseur variable tout en maintenant un alignement homéotrope des couches. La réponse élastique de ce réseau rend compte qualıtatıvement des différentes caractéristiques du profil de force mesuré et permet de préciser la forme parabolique des oscillations. L'analyse des données produit alors une évaluation 'du module de compressibilité de couche avec une bonne précision. Cependant cette analyse élastique n'explique pas pourquoi les oscillations se superposent à une attraction aux faibles séparations. Nous dıscutons deux origines possibles pour cette attraction Une comparaison quantitative ne permet pas de conclure sur leur pertinence.

\begin{abstract}
The lamellar phase of a lyotropic solution in a confined geometry is investigated with a surface force apparatus (SFA). The measured interaction between the two SFA surfaces immersed in the mesophase is periodic, oscillatory, decaying, and discontinuous. A model is developed to account for the specific confining geometry of the SFA It is based on an array of concentric dislocation loops that allows a homeotropic alignment of the layers in a space of variable thickness. The elastic response of the array at any strain accounts for the periodicity and the oscillatory nature of the force profile and also describes the parabolic shape of the oscillations The layer compressibility modulus can be extracted from the data with a good accuracy. However the elastic analysis fails to explain the attractive background on which the oscillations are superimposed at small separations Two possible origins for the attraction are discussed, but unfortunately a quantitative comparison does not allow a definitive conclusion on the actual cause.
\end{abstract}




\section{Introduction}

The interactions between two surfaces immersed in isotropic media have been extensively studied over recent years [1]. Their knowledge and understanding are of fundamental importance especially for the science of colloids [2]. Experimentally, a lot of attention has been paid to the ubiquitous van der Waals dispersion forces [3-7], electrical double layer interactions [8-11], steric repulsions (in polymer solutions for instance) [12,13], depletion attractions [14-16], and structural forces in simple fluids [17-19] and micellar solutions $[15,20]$.

Comparatively much less attention has been devoted to the interactions between two surfaces in anisotropic fluids such as liquid crystals [21-27]. Because of their long-range organization, liquid crystals are expected to generate interactions between surfaces over macroscopic distances. Nematic liquid crystals, for example, can transmit torques [28] via twist deformations. In the case of a lamellar (or smectic) phase sandwiched between two rigid surfaces with homeotropic alignment (i.e., the optic axis is perpendicular to the surfaces) three additional interactions are expected.

The most intuitive has an elastic origin: every change in the separation between two walls would generate a strain field on the structure and, for weak distorsions, the liquid crystal will respond elastically giving rise to an interaction between the surfaces. The sign of the interaction depends on whether the medium is compressed or dilated, on the alignment induced by the walls, and on the geometry of the confinement gap as will be discussed later.

The other two interactions are more subtle. One is caused by the enhancement of the order parameter near each surface over a distance of the order of a smectic coherence length. Below some separation these boundary layers overlap and the excess surface free energy becomes a function of the separation. Under symmetric boundary conditions, the gradient of the order parameter profile decreases as the surfaces approach each other, thereby the free energy decreases as well, and the resulting interaction is attractive [29]. Finally, there is a third contribution arising from orientational fluctuations in anisotropic mesophases [30,31]. Consider again the case of a smectic slab confined between two hard walls with a homeotropic alignment. The rigid boundary conditions suppress a number of fluctuating modes, namely those having a lateral extension larger than the separation between the walls. The free energy associated with this "depletion of modes" again decreases with decreasing separation and the interaction is attractive. A remarkable feature is that the attraction decays more slowly than the van der Waals interaction and may become dominant at large separations [30].

Recently, we reported force measurements between two surfaces immersed in a lamellar mesophase as a function of their separation [24]. We showed how the compressibility modulus of the layered stack could be extracted from the measured data. The present article completes the latter work and aims to provide a full theoretical background. Using a model that describes the array of edge dislocation loops arising from the experimental wedge-shaped geometry, the ever repulsive nature of the measured elastic forces is explained and a full justification of our previous derivation [24] of the elastic modulus is given. Furthermore, the experimental observation of a long-range attractive backgound is discussed in view of the additional two contributions to the total force mentioned above.

The article is organized as follows: the experimental observations are first recalled in Section 2 and the edge dislocation loop model is introduced in Section 3. Both elastic and nonelastic responses to an external strain are calculated. In Section 4, comparison between the predictions of the elastic theory with the experimental data is carried out and some quantitative conclusions about the compressibility modulus are derived. In a further step, we examine the other interactions arising from the enhancement of ordering and from the restriction of fluctuating modes in the peculiar wedge-shape of the experimental confining geometry. Finally, 
Section 5 is devoted to concluding remarks.

\section{Experimental Observations}

The water-swollen lamellar phase studied was made of sodium dodecyl sulfate (SDS), 1pentanol, and water. The SDS (from BDH, extra pure) was purified according to the method described in reference [32]. Pentanol (from Sigma) was distilled once before use and the water was deionized and doubly distilled. The samples were prepared by weighing the components and mixing them by repeated centrifugation (compositions by weight: $7.17 \%$ SDS, $17.48 \%$ pentanol, and $75.35 \%$ water). The interlamellar spacing was checked by X-ray diffraction and found to be equal to $8.8 \pm 0.1 \mathrm{~nm}$.

A surface forces apparatus $[1,8]$ was used to probe the interactions between mica surfaces immersed in the lamellar phase. This instrument is capable of measuring the separation between two surfaces of molecularly smooth mica sheets mounted in a crossed-cylinder configuration, with an accuracy of $\pm 0.2 \mathrm{~nm}$ and the force, $F$, with a resolution of $10^{-7} \mathrm{~N}$ [33]. When normalised with the mean radius of curvature of the surfaces, $R(R \approx 1-2 \mathrm{~cm})$, the forces are uncertain to about $10 \%$, as the error in the absolute scale of $F / R$ is mainly dictated by the accuracy in the determination of $R$. The smallest normalised force that can be detected is within $0.01 \mathrm{mN} / \mathrm{m}$. The mica surfaces were totally immersed in the lyotropic liquid crystal. The system was allowed to equilibrate for at least $24 \mathrm{~h}$ at a separation of a few micrometers. The optical interference technique [34] employed in these experiments allows us to measure not only the thickness of the liquid crystal film but also its average refractive index $(1.335 \pm 0.010$ over nearly the whole range of surface separations except at small separations where it is $1.33 \pm 0.02$ below $2.8 \mathrm{~nm}$ ). The interference fringes are split into linearly polarized components by the birefringent micas. When the surfaces are in contact, this gives the net birefringence of the two mica sheets [34]. When the surfaces are separated in the lamellar phase, the fringe splitting remains constant, indicating that the birefringent liquid crystal keeps a homeotropic orientation up to large separations. This behavior is in agreement with prior observation between crossed polarizers under a microscope. Homeotropic alignment was obtained within a few hours. All experiments were performed at $22.0 \pm 0.1^{\circ} \mathrm{C}$.

The normalized force, $F / R$, measured between the two mica surfaces is presented as a function of separation in Figure 1. At large separations no force is detected within the sensitivity of the apparatus. As the separation is reduced, the force oscillates irregularly with surface separation between 1000 and $500 \mathrm{~nm}$. At smaller separations the periodicity becomes regular (Fig. 1) and correlates well with the reticular spacing, $d$, measured by $\mathrm{X}$-ray scattering in a bulk sample. This spatially decaying oscillatory force is observed down to contact, with the period $d$ (separation between adjacent minima, Fig. 1b) remaining constant. The last 16 oscillations before contact, shown in Figure 1b, highly reproducible, were tracked with both inward (compression) and outward (dilation) runs using the prezoelectric tube of the apparatus. The force-distance profile appears discontinuous because of the intrinsic mechanical instability of the cantilever that supports one of the mica surfaces: when the gradient of the force is larger than the spring constant $K$ (i.e., $\partial(F / R) / \partial D>K / R$ ) the system jumps from unstable to stable regimes, leaving unexplored and inaccessible regions [8]. The inward jump from one stable position to the next occurs within a few seconds. As shown in Figure 1b, the force-distance profile consists of successive parts, each of which is non-linear in shape. Both their magnitude and consequently their slope increase as the surface separation decreases.

The oscillations are superimposed on a net long-range attractive background force (Fig. 1a), which starts at about the $20^{\text {th }}$ oscillation $(\approx 200 \mathrm{~nm})$. The magnitude of the attraction increases with decreasing surface separation until it finally turns around and becomes repulsive 


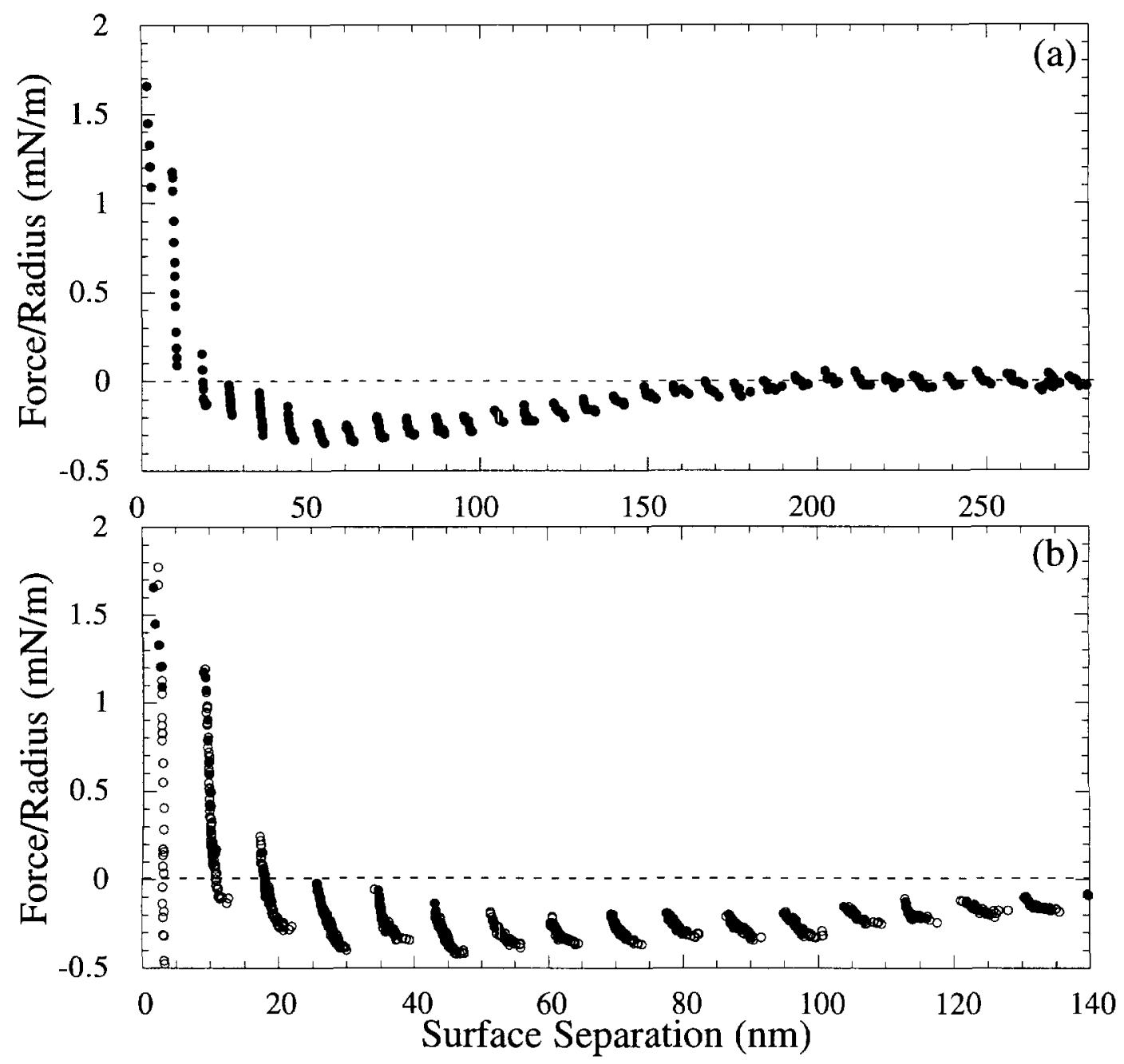

Fig. 1 - The force $F$ scaled by the radius of curvature $R$ of the curved mica surfaces immersed in a swollen lamellar mesophase (sodium dodecyl sulfate, water, and 1-pentanol) as a function of separation. The upper plot (a) shows the force measured with the surfaces approaching each other. The lower plot (b) shows an enlargement of the data in (a) (filled symbols) together with the outward (open symbols) force runs for the first 16 oscillations

at small separations $(\approx 20 \mathrm{~nm}$ ). At the onset of the contact, no hard wall behaviour is observed and measurement of the refractive index $(1.33 \pm 0.02)$ of the medium confined between the mica surfaces at separations smaller than $2.8 \mathrm{~nm}$, seems to indicate that no surfactant layers are present. This is to be expected since the anionic SDS does not adsorb on the negatively charged mica surfaces in aqueous solutions [35]. We therefore conclude that a water layer coats each wall before the surfactant bilayers and water layers get alternately stacked. 


\section{Theoretical Approach}

Let us first recall what is expected when an external strain is applied to a smectic mesophase confined between two parallel flat plates with "rigid" boundary conditions (i.e., smectic layers parallel to the plates). Pershan and Prost have discussed [36] the relaxation of the induced elastic stress by edge dislocation loop with Burgers vector of unit strength. They have considered the case of a perfect smectic sample, initially free of any defects, confined between two hard flat walls a distance $e$ apart, where $e$ is an integral multiple of the equilibrium layer thickness at zero stress, $d$. A tensile or compressive force applied onto one boundary induces a strain $\delta e / e$. As long as $|\delta e|<d / 2$, edge dislocation loops are energetically unfavorable. If $|\delta e|>d / 2$, the elastic energy of the system is lowered by the introduction or the loss of a layer depending on the sign of the strain. Under a compressive applied force, the reduction of one layer would be achieved by the nucleation of an edge dislocation loop inside the confined sample (i.e., not too near the boundaries and more likely in the vicinity of the medium plane). Pores, or edge dislocation loops of unit Burgers vector can be thermally activated in the layers. For any strain $\delta e>d / 2$, there exists a critical pore radius, $r_{\mathrm{c}}$, above which the loop will grow unstably throughout the sample thus reducing the number of confined layers by one. If $r<r_{\mathrm{c}}$, the loop will shrink spontaneously. This nucleation process is expected to follow a Boltzmann distribution: the nucleation rate per unit volume of stable loops is $f \sim \exp \left(-E_{\mathrm{c}} / k_{\mathrm{B}} T\right)$ in which $E_{\mathrm{c}}$ is the energy barrier of the loop to overcome $r_{\mathrm{c}}$.

For practical reasons, the surface force apparatus uses curved surfaces in a crossed-cylinder geometry [8]. The above analysis is for flat plates and needs to be adapted. The vertical separation, $h$, of the two surfaces simply reads:

$$
h(r, \theta)=e+\frac{r^{2}}{2 R}+O\left(\left(\frac{r}{R}\right)^{4}, \theta\right)
$$

where $(r, \theta)$ are the cylindrical coordinates in the plane parallel to the axes of the two cylinders and $e$ is the thickness at center (closest separation of the two cylinders). If terms of order higher than two in $r / R$ are neglected (in the reported experiments $e / R<10^{4}$ ) the angular coordinate $\theta$ is irrelevant and the crossed-cylinder geometry is equivalent to a sphere of radius $R$ near a flat surface.

Experimental observations show that the sample tends to orient homeotropically (i.e., with layers parallel to the walls). In a confined medium of non-uniform thickness, such as a sphere against a flat plane, there is a conflict between the homeotropic alignment and the need for a constant layer spacing. as a result defects must arise. Thus for a wedge-shaped geometry, an array of edge dislocation loops is expected and has been observed [37,38]. Although the preferred array may not always be made of circular dislocations with Burgers vectors of unit strength $(b=1)$ [39], our experimental data strongly suggest this is the case at short separations. Therefore in the following analysis, $b=1$ is assumed for all separations (see also discussion in Section 4).

To begin with, the space in which the confined sample exists can be divided into $N$ adjacent torus-like cells, Figure 2. Each cell of rank $\imath$ is delimited by an inner radius, $\rho_{\imath}$, where the thickness, $h\left(\rho_{\imath}\right)$, is an integral multiple of the reticular distance: $h\left(\rho_{\imath}\right)=n_{\imath} d$, and an outer radius, $\rho_{\imath+1}$, where the thickness is increased by one layer period: $h\left(\rho_{2+1}\right)=\left(n_{\imath+1}\right) d$. In this picture every cell of rank $\imath>0$ contains one edge dislocation loop located at radius $r_{\imath}$ as sketched in Figure 2. For the central cell $\imath=0$, the inner radius is zero. Whether it contains a dislocation or not depends on thickness at center $e$. To good approximation, the cells are considered to be independent, i.e., two adjacent loops do not interact. Indeed, it is easy to check that the strain fields due to the presence of dislocations do not overlap. According to 


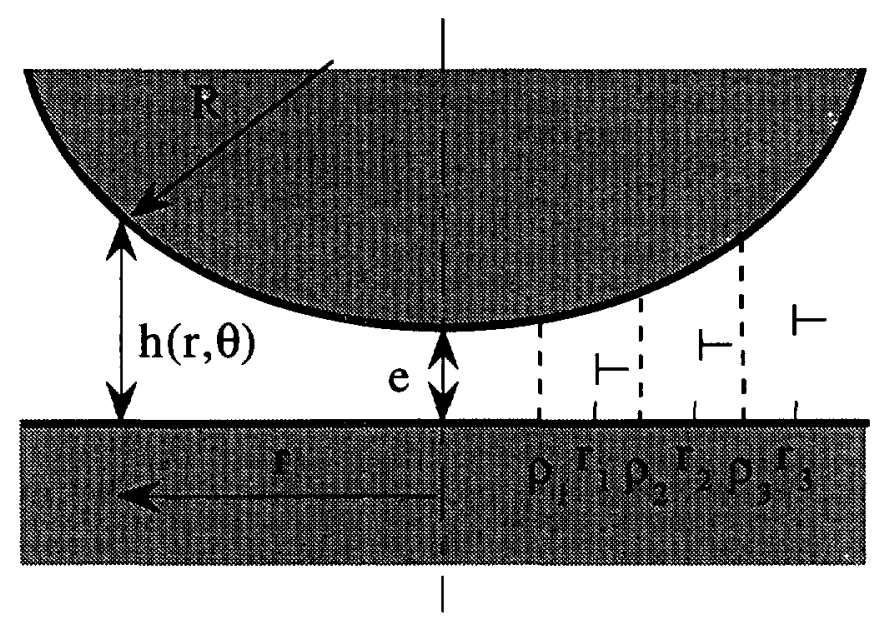

Fig. 2. - Schematic diagram showing a lamellar mesophase confined between a sphere of radius $R$ and a flat plane An array of concentric dislocation loops is introduced in order to fill up the confining space of varying thickness under homeotropic alignment. The sample is arbitrarily divided into $N$ adjacent torus-like cells. Each cell is delimited by an inner radius, $\rho_{\imath}$, where the thickness, $h\left(\rho_{\imath}\right)$, is an integral multıple of the reticular distance: $h\left(\rho_{\imath}\right)=n_{\imath} d$, and an outer radius, $\rho_{\imath+1}$, where the thickness is increased by one layer period: $h\left(\rho_{\imath+1}\right)=\left(n_{\imath}+1\right) d$. In this picture every cell of rank $\imath>0$ contains one edge dislocation loop of radius $\tilde{r}_{\imath}$. For the central cell $\imath=0$, the inner radius is zero. Whether it contains a dislocation or not depends on thickness at center $e$.

de Gennes $[28,40]$ the strain field decays as $\exp \left(-x^{2} /|z| \lambda\right)$, where $\lambda$ is the penetration length and $x$ and $z$ are coordinates parallel and perpendicular to the layers, respectively. The strain field is negligible outside the parabola $x^{2}=\lambda|z|$. Because of the characteristics of the surface force apparatus $(R \approx 1-2 \mathrm{~cm}$ ), the distance between two adjacent dislocations appears to be a few micrometers whereas the range of the strain field, like the penetration length, is only of the order of a few reticular distances, i.e., $\approx 10 \mathrm{~nm}$. Each loop can thus be considered as isolated and the energy of the array of dislocations, $E_{T}$, may now be obtained as the sum of the contributions from each cell, $E_{\imath}: E_{T}=\sum_{\imath=0}^{N_{\max }} E_{\imath}$, where the upper boundary, $N_{\max }$, is defined by the sample thickness at the edge of the mica surfaces, typically about $1-2 \mathrm{~mm}$.

Following Nallet and Prost [39] the different elastic contributions in each cell are now evaluated. The cell $\imath=0$ plays a special role and will be treated separately. Three contributions should be retained for every cell $\imath>0$ :

i) The first one is the wedge confinement energy, $E_{\mathrm{W}_{2}}$, due to the elastic distorsion of the smectic material [41]. It originates from the strain field outside the parabola mentioned above. Over the region spanning from the inner radius, $\rho_{\imath}$, to the dislocation loop radius, $r_{\imath}$, the smectic layers are stretched, whereas from the loop radius, $r_{\imath}$, to the outer radius, $\rho_{\imath+1}$, the layers are compressed. Hence:

$$
E_{\mathrm{W}_{\imath}}=\frac{B}{2}\left[\int_{\rho_{\imath}}^{r_{\imath}} 2 \pi r \mathrm{~d} r \int_{0}^{h_{\imath}}\left(\frac{h(r)-h_{\imath}}{h_{\imath}}\right)^{2} \mathrm{~d} z+\int_{r_{\imath}}^{\rho_{\imath+1}} 2 \pi r \mathrm{~d} r \int_{0}^{h_{\imath+1}}\left(\frac{h(r)-h_{\imath+1}}{h_{\imath+1}}\right)^{2} \mathrm{~d} z\right]
$$

where $B$ is the compression modulus, $h_{\imath}=h\left(\rho_{\imath}\right)=n_{\imath} d$ and $h_{\imath+1}=h\left(\rho_{\imath+1}\right)=\left(n_{\imath}+1\right) d$ are 
the thicknesses of the unstrained material for $\rho_{\imath}<r<r_{\imath}$ and $r_{\imath}<r<\rho_{\imath+1}$, respectively.

i1) The second contribution arises from the distorsion field around the dislocation core, inside the parabola of strain [28]. According to Kléman, this far-field energy, $E_{\mathrm{F}_{\mathrm{s}}}$, reads [42]:

$$
\begin{aligned}
E_{\mathbf{F}_{z}} & =2 \pi r_{2} \gamma \\
\gamma & =\frac{B \lambda d}{2}
\end{aligned}
$$

where $\lambda$ is the penetration length, $\lambda^{2}=K / B$, and $K$ the bending elastic modulus.

One should keep in mind that the above equations have been established for an infinite medium or for dislocation loops far enough from any interface. For the present confined system, when the number of smectic layers is small at the center of the cell, i.e., when the separation is smaller or comparable to $l$, the defects interact with the walls, therefore a separation dependence of $g$ is now expected, $g=g(e)$. Later on, in the discussion section, we will mention a feature of the experimental force profile which might be related to this dependence.

iii) The third contribution originates from the core of the defect itself. For a dislocation of small Burgers vector, like a pore in a membrane, the core energy cannot be described by a continuum theory and a microscopic approach is more appropriate [42]. To first approximation, the core energy, $E_{\mathrm{C}_{2}}$, can be considered as a linear function of the pore radius:

$$
E_{\mathrm{C}_{2}}=2 \pi r_{2} \gamma^{\prime}
$$

where the phenomenological parameter, $\gamma^{\prime}\left(\gamma^{\prime}>0\right)$, is an energy per unit length.

The net elastic energy, $E_{\imath}$, stored in each cell $\imath$ is then:

$$
E_{\imath}=E_{\mathrm{W}_{\imath}}+E_{\mathrm{F}_{\imath}}+E_{\mathrm{C}_{\imath}}
$$

Minimizing equation (5) with respect to $r_{\imath}$ gives the equilibrium radius $\tilde{r}_{2}$ of the dislocation loop:

$$
\tilde{r}_{\imath} \frac{B}{2}\left[h_{\imath}\left(\frac{h\left(\tilde{r}_{\imath}\right)-h_{\imath}}{h_{\imath}}\right)^{2}-h_{\imath+1}\left(\frac{h\left(\tilde{r}_{\imath}\right)-h_{\imath+1}}{h_{\imath+1}}\right)^{2}\right]+\gamma+\gamma^{\prime}=0
$$

The next two steps are to calculate the energy of the whole array of dislocations, $E_{T}=$ $E_{0}+\sum_{\imath=1}^{N_{\max }} E_{\imath}\left(\tilde{r}_{\imath}\right)$, knowing $\tilde{r}_{\imath}$ from equation (6), and then to determine the net interaction, $F_{T}$, between the plane and the sphere:

$$
F_{T}(e)=-\frac{\mathrm{d} E_{T}}{\mathrm{~d} e}
$$

In order to understand the contribution of each term in equation (5), we shall first forget the two line energies $E_{\mathrm{F}_{2}}$ and $E_{\mathrm{C}_{2}}$ (i.e. set $\gamma+\gamma^{\prime}=0$ in equation (6)). The equilibrium radius (noted $\vec{r}_{2}$ in this case) is then given by:

$$
\frac{\bar{r}_{\imath}^{2}}{2 R}+e=\sqrt{n_{\imath}\left(n_{\imath}+1\right)} d
$$

Hence, circular edge dislocations remain located on planes of constant elevation $h\left(\bar{r}_{\imath}\right)=$ $\sqrt{n_{\imath}\left(n_{\imath}+1\right)} d$ when the separation at center, $e$, varies, and the local reticular distance of 
the array decreases as the radius increases $\left(\overline{r_{\imath+1}}-\overline{r_{\imath}} \cong \sqrt{\frac{R d}{2 n_{\imath}}}\right.$ for large radius $\left.\overline{r_{\imath}}\right)$. The elastic energy in each cell, $E_{\imath}=E_{w_{\imath}}$ is readily calculated, combining equations (1) and (8):

$$
\begin{aligned}
E_{\imath>0}= & \pi R d^{2} B\left[\left(\frac{4 n_{\imath}+1}{3} \sqrt{n_{\imath}\left(n_{\imath}+1\right)}-n_{\imath} \frac{4 n_{\imath}+3}{3}\right)+\right. \\
& \left.\left(\left(n_{\imath}+1\right) \frac{4 n_{\imath}+1}{3}-\frac{4 n_{\imath}+3}{3} \sqrt{n_{\imath}\left(n_{\imath}+1\right)}\right)\right]
\end{aligned}
$$

The cells of rank $\imath>0$ do not contribute to the interaction between the surfaces since the elastic energy $E_{2}>0$ does not depend on separation at center $e$. In other words, the attraction of the stretched areas exactly cancels the repulsion due to the compressed areas. This important result can be demonstrated in a more general way (see Appendix A).

We have now to consider the central cell $\imath=0$ which extends from center $(r=0)$ up to a radius $\rho_{1}$ where the thickness $h\left(\rho_{1}\right)$ equals $n_{1} d$, the lowest possible integral multiple of $d$. The free energy in this cell can be integrated either with or without a dislocation loop from equation (2). The excess elastic free energy reads:

$$
\Delta E_{0}=E_{w_{0}}^{\text {with }}-E_{w_{0}}^{\text {without }}=\frac{\pi d B}{24 e_{0}^{2} R^{2}}\left[r_{0}^{6}+6 \operatorname{Rer}_{0}^{4}+12 R^{2}\left(e^{2}-e_{0}^{2}\right) r_{0}^{2}\right]
$$

where $e_{0}=e_{0}\left(n_{1}\right)=\sqrt{n_{1}\left(n_{1}-1\right)} d$. Equation (10) indicates that a continuous transition occurs when the quadratic term changes sign. As long as the thickness at center $e$ is larger than $e_{0}$, the quadratic term is positive and the excess energy is minimum for $\bar{r}_{0}=0$, i.e., no dislocation is present in the central cell $\left(n_{0}=n_{1}\right)$. The layers are compressed over the whole cell and the net interaction is repulsive. When the separation between the walls is reduced, as soon as $e<e_{0}$, the free energy exhibits a negative minimum at the equilibrium radius $\bar{r}_{0}$ given by:

$$
\bar{r}_{0}=\sqrt{2 R\left(e_{0}-e\right)}
$$

and $n_{0}=n_{1}-1$. Eventually, the loops expand continuously when the surfaces are approached. A new loop appears each time the thickness at center $e$ crosses a value $e_{0}\left(n_{1}\right)=\sqrt{n_{1}\left(n_{1}-1\right)} d$ in which $n_{1}$ is an integer. Conversely, the dislocation loops shrink and disappear when the surfaces are pulled apart. That is application of an external strain, dilating the central cell (initially a perfect smectic slab with no dislocation) would cause dislocations to move into it from the sides in order to reduce the net external force on the surface to zero. The process in which a slight change in sample thickness produces a uniform force tending to move the dislocation through the sample, thereby relaxing the applied stress, is in agreement with Pershan's description [41]. In the absence of localized centers that might pin them, edge dislocations will always move in layered systems in such a way as to exclude stress from the materials. As noted by Pershan, in thin smectic samples $(e<1 \mu \mathrm{m})$ with rigid boundary conditions (without surface tension effect [43]), appearance or annihilation of dislocations will be largely confined to the central position [41].

The elastic energy of the central cell depends explicitly on the sample thickness at center $e$, and remarkably the total elastic interaction between the two surfaces arises exclusively from this cell. The elastic force reads:

$$
F_{T}(e)=\pi R B \frac{\left(e-n_{0} d\right)^{2}}{n_{0} d}=2 \pi R f_{T}
$$

in which $f_{T}$ is the compression elastic energy per unit area of $n_{0}$ layers of a smectic material homeotropically aligned between two flat plates a distance $e$ apart. Equation (12) turns out 
to identify with the classical Derjaguin equivalence, which relates the force between a sphere against a plate scaled by the radius of curvature to the density of interaction free energy between two parallel plates [44]. The applicability of the Derjaguin approximation was not expected to hold as the elastic potential $f_{T}$ is long-range. The reason lies obviously in the exact vanishing of the interaction for all cells of rank $\imath>0$ resulting from the regular array of dislocation loops.

We first note that the elastic interaction $F_{T}(e)$ strictly vanishes each time the separation at center $e$ is an integral multiple of $d$, the equilibrium layer thickness at zero stress. Moreover, the elastic contribution is always repulsive, whatever the sign of the applied strain. The forcedistance profile is a set of parabolae, each one corresponding to a different number of layers $n_{0}$ at center, as illustrated in Figure 3a. Two adjacent parabolae intercept at the critical thicknesses $e_{0}\left(n_{0}\right)=\sqrt{n_{0}\left(n_{0}-1\right)} d$ for which the first loop of the array is either nucleated or annihilated. At thermodynamic equilibrium the force profile is fairly periodic, the separation between two adjacent intersects rapidly approaching the reticular distance $d$ as $n_{0}$ increases. The amplitude of the oscillations, and accordingly their slope, increases as the separation between the two surfaces decreases (Fig. 3a). The minima of the parabolae line up on a baseline $F_{T}=0$.

The experimental data (Fig. 1) actually exhibit a very similar oscillatory force profile except that the baseline (i.e., the envelope on which lie the minima of each oscillation) clearly forms a long-range attractive background. Before proceeding further in the comparison, we need to consider how the elastic force (Eq. (12)) is affected by the line energies, i.e., the far-field energy (Eq. (3)) and the core energy (Eq. (4)).

It is intuitive that the line tensions will reduce the equilibrium radius (Eq. (8)) in each cell, i.e., $\tilde{r}_{\imath}<\bar{r}_{\imath}$ Consequently, all the elastic energies $E_{\imath}\left(\tilde{r}_{\imath}\right)$ are expected to depend explicitly on $e$ and every cell of rank $\imath$ will now contribute to the net interaction between the two surfaces. The force (Eq. (7)) can be rewritten as:

$$
F_{T}(e)=\sum_{\imath=0}^{N_{\max }} \frac{\partial E_{\imath}}{\partial e}+\frac{\partial E_{\imath}}{\partial r_{\imath}} \frac{\mathrm{d} r_{\imath}}{\mathrm{d} e}
$$

The Euler-Lagrange condition imposes $\partial E_{\imath} / \partial r_{\imath}$. With the definition of the wedge elastic energy (Eq. (1)) (since $\partial E_{\imath} / \partial e E_{w_{\imath}} / \partial e$ ) it is found that:

$$
\frac{\partial E_{\imath}}{\partial e}=\pi R B\left[h_{\imath}\left(\frac{h\left(\tilde{r}_{\imath}\right)-h_{\imath}}{h_{\imath}}\right)^{2}-h_{\imath+1}\left(\frac{h\left(\tilde{r}_{\imath}\right)-h_{\imath+1}}{h_{\imath+1}}\right)^{2}\right]
$$

The contribution of every loop is simply obtained by combining equations (6) and (14) (see also Appendix A):

$$
\frac{\partial E_{2}}{\partial e}=-\frac{2 \pi R}{\tilde{r}_{2}}\left(\gamma+\gamma^{\prime}\right)
$$

Finally consıdering the special role of the central cell as previously, the total elastic force between the surfaces is:

$$
F_{T}(e)=\pi R B \frac{\left(e-n_{0} d\right)^{2}}{n_{0} d}+2 \pi R\left(\gamma+\gamma^{\prime}\right) \sum_{0}^{N_{\max }} \frac{1}{\tilde{r}_{\imath}}
$$

The new expression of the force deviates from the Derjaguin equivalence (Eq. (12)): the fortunate cancellation of the interaction over the cells of rank $\imath>0$ no longer occurs when the line energies are taken into account (see also Appendix A). An additional interaction proportional to the line energies $\gamma+\gamma^{\prime}$ is now superimposed on the Derjaguin form (Eq. (12)). 

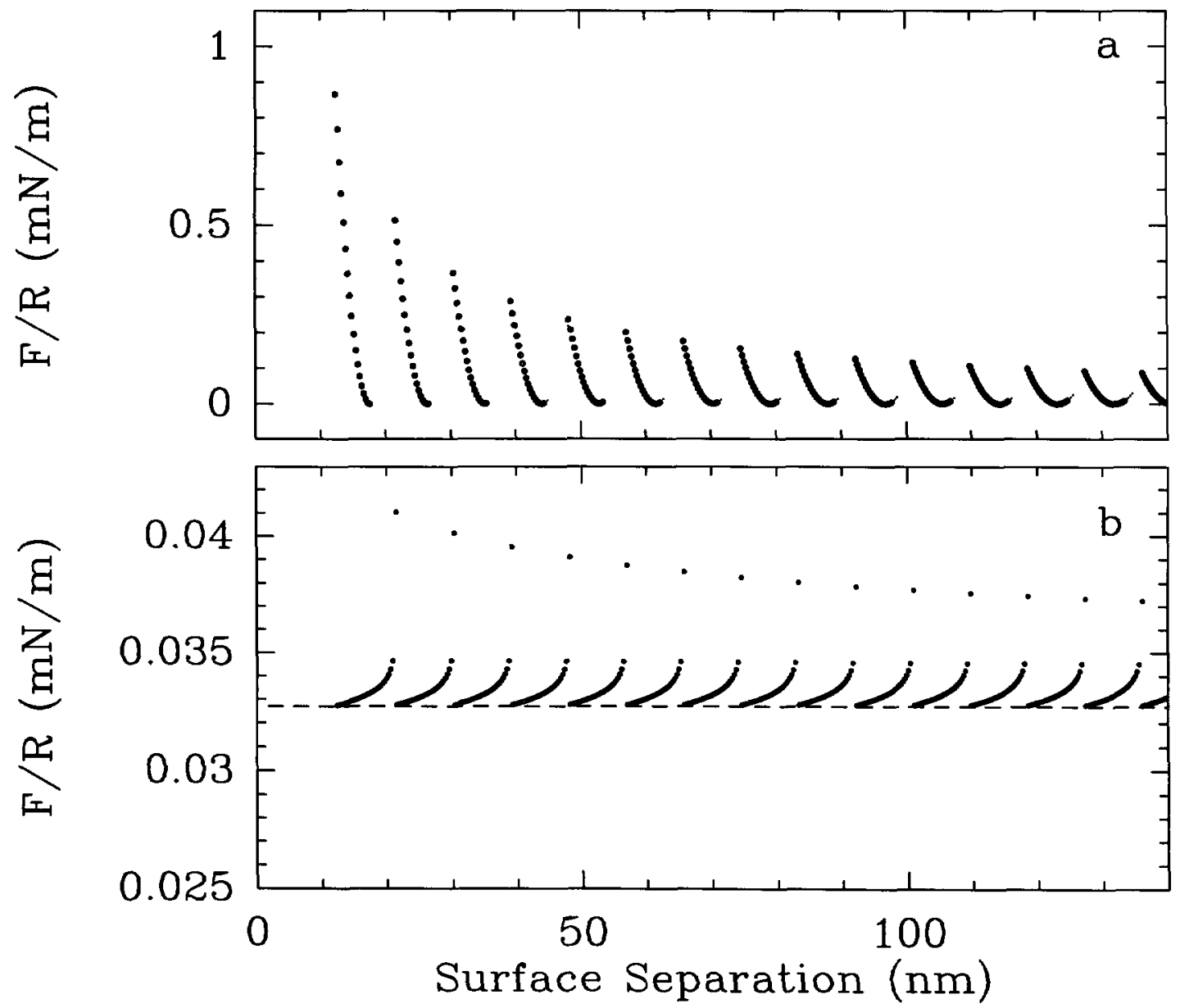

Fig 3. - Theoretical elastic force profile between a sphere of radius $R$ and a plane confining a smectic phase of layer spacing $d$ when an array of concentric elementary dislocation loops allows an homeotropic alignment of the mesophase Numerical minumizations have been performed to obtain the loop radius at each separation. The parameters retained for the simulation were, $R=2 \mathrm{~cm}, B=10^{5}$ $\mathrm{J} / \mathrm{m}^{3}$ (layer elastic modulus), $d=8.8 \mathrm{~nm}, \tilde{\lambda}=2 \mathrm{~nm}$ (effective penetration length) and $N_{\max }=50$ (number of loops considered in the array). The upper plot (a) corresponds to the Derjaguin term of equations (12) and (16) when the line tension of loops are omitted. In the lower plot (b) the filled symbols are the complementary discontinuous contribution arising from the line tension expressed by the sum term in equation (16). The net elastic force is the sum of the two plots. In (b) the dotted baseline is the numerical result given by equation (21) The intrinsic instability of the SFA (see Section 2) has been mimicked in (a) by representing the unaccessible regions with light symbols (the actual spring constant of the device was compared to the gradient of the calculated force).

This new term is always positive (i.e., repulsive) at all separations. Its exact magnitude is hard to estimate as it results from the summation of a slowly decaying function $\left(1 / \tilde{r}_{\imath}\right)$ up to large values of $\imath$ where some of the assumptions made previously are no longer valid, namely $r \ll R$ and Burgers vectors remaining small (here being elementary) [39]. We postpone this estimation to the next section. 
From a dynamical point of view, the integration of line energies introduces a significant qualitative difference. Consider again the central cell and include the far-field and the core energy contributions into the excess elastic energy. Equation (10) now contains an additional positive linear term:

$$
\Delta E_{0}=\frac{\pi d B}{24 e_{0}^{2} R^{2}}\left[r_{0}^{6}+6 \operatorname{Rer}_{0}^{4}+12 R^{2}\left(e^{2}-e_{0}^{2}\right) r_{0}^{2}+24 e_{0}^{2} R^{2} \tilde{\lambda} r_{0}\right]
$$

where $\tilde{\lambda}=2\left(\gamma+\gamma^{\prime}\right) / B d$ has the dimension of a length (it would be the penetration length if the core energy were ignored). The emergence of a new loop would still be driven by the applied strain but the nature of the transition is now first-order-like. The critical thickness, $e_{0}$, is shifted towards a lower separation $e_{\mathrm{c}}=e_{0}-e$. For the experimentally relevant limit $r^{2} \ll R e$ the shift can be estimated to first order as:

$$
\epsilon=e_{0}-e_{\mathrm{c}} \cong \frac{3}{2}\left(\frac{\tilde{\lambda}^{2} e_{0}^{2}}{2 R}\right)^{1 / 3}
$$

The situation is similar to the Pershan-Prost study [36]. For $e<e_{\mathrm{c}}$ the excess free energy, $\Delta E_{0}$, exhibits two minima: a metastable minimum at $r=0$ and a negative one at $\tilde{r_{0}}$ separated by an activation barrier $\Delta E_{0}\left(r_{c}\right)$. Thus $r_{c}$ is a critical radius beyond which a thermally activated pore will grow spontaneously to reach the equilibrium radius $\tilde{r_{0}}$. To first order, the critical radii and the equilibrium radil read:

$$
\begin{aligned}
& \frac{r_{\mathrm{c}}^{2}}{2 R} \cong \frac{2-\sqrt{3}}{3} \epsilon \\
& \frac{\tilde{r}_{0}^{2}}{2 R} \cong \frac{2}{3} \epsilon
\end{aligned}
$$

The nucleation of the pore will occur at the critical separation $e_{\mathrm{c}}$ in a reasonable time, if the activation barrier $\Delta E_{0}\left(r_{\mathrm{c}}\right)$ is not too large compared to $k_{\mathrm{B}} T$. Otherwise, no dislocation can be initiated, and a metastable state will be built. The system will remain in this state even at separations lower than $e_{\mathrm{c}}$ until the height of the activation barrier is lowered enough in applying a further strain to allow the nucleation to be initiated. To first order, the energy barrier at $e_{\mathrm{c}}$ can be evaluated as:

$$
\Delta E_{0}\left(r_{\mathrm{c}}\right) \cong \frac{2 \sqrt{3}-3}{3} \frac{\pi R B d}{e_{0}} \epsilon^{2}
$$

To conclude this paragraph devoted to the elastic behaviour of a lamellar phase under strain between a sphere and a plane, the results can be summarized as follows. When the applied strain $|\delta e|$ is lower than some threshold strain $\alpha d / 2$ ( $\alpha$ being slightly larger than 1) the liquid crystal responds elastically, giving rise to an effective interaction between the two surfaces that is always repulsive. Beyond some threshold strain, a dislocation loop nucleates and reduces the experienced stress. If the activation barrier is not too high, successive nucleations can occur giving rise to a periodic force-distance profile when the two confining surfaces are moved.

\section{Discussion and Analysis}

As mentioned above, taking into account the elastıc contributions only (Eq. (16)), is not enough to describe the experimental force-distance profile as a whole. Certainly, these elastic 
contributions describe the observed parabolic oscillations quite well, but they do not generate any attractive long-range background. Non-elastic contributions such as a local enhancement of the smectic order parameter in the vicinity of the walls and the restriction of some fluctuations modes by confinement must also be considered. Before analysing these effects in detail, it is worth comparing the effects of the two terms involved in the elastic force (Eq. (16)). For the lyotropic system considered here, and for the experimental conditions reported above, the first term given directly by the Derjaguin approximation is dominant. Perturbations arising from the second term are either below the apparatus sensitivity or below the spacial resolution.

4.1. Elastic Terms. - Let us consider the line energy contribution to the elastic force expressed by the summation term in equation (16). The first term $(\imath=0)$ of the series is the largest one when it exists, i.e., when the center thickness is stretched $\left(e>n_{0} d\right)$. We will again consider it separately. We begin by estimating the summation from $\imath=1$ to $N_{\max }$.

A straightforward calculation shows that $\tilde{r}_{\imath}^{2} / 2 R+e \cong e_{2}\left(1-\tilde{\lambda} / 2 \bar{r}_{2}\right)$ where $e_{\imath}=\left(n_{\imath} n_{2+1}\right)^{1 / 2} d$ and $\vec{r}_{\imath}$, calculated at zero line tension $(\tilde{\lambda}=0)$, is defined in equation (8). For $\imath>0$, the order of magnitude of $\bar{r}_{2}$ is always larger than a few micrometers $(R \cong 2 \mathrm{~cm} ; d=8.8 \mathrm{~nm})$ whereas $\tilde{\lambda}$ is comparable to the reticular distance $d$, say a few tens of nanometers. Thus $\tilde{r}_{\imath}$ is very close to $\bar{r}_{\imath}\left(\tilde{r}_{\imath} \cong \bar{r}_{\imath}\right)$, which means that the dislocation loop is virtually unaffected by the line tension $\gamma+\gamma^{\prime}$ In other words, the confining effect alone determines the equilibrium radius provided that $R \gg d$ (as in these experiments). A further approximation is to note that $\left(n_{\imath} n_{\imath+1}\right)^{1 / 2} \cong n_{\imath}+1 / 2$. According to the loop array model the central thickness $e$ can then vary grossly from $\left(n_{0}-1 / 2\right) d$ to $\left(n_{0}+1 / 2\right) d$ without any change of the number of layers at the center, $n_{0}$. Then the sum $\sum_{i=1}^{N_{\max }} \tilde{r}_{i}^{-1}$ is bounded by $(2 R d)^{1 / 2} \sum_{\imath=2}^{N_{\max }+1} \imath^{-1 / 2}$ and $(2 R d)^{1 / 2} \sum_{i=1}^{N_{\max }} \imath^{-1 / 2}$. Using:

$$
\sum_{\imath=\jmath>0}^{N} \imath^{-1 / 2} \cong \int_{\jmath-1}^{N}(2 x+1)^{-1 / 2} \mathrm{~d} x
$$

since $N_{\max }$ is large one deduces that $\sum_{\imath=1}{\tilde{r_{\imath}}}^{-1}$ is almost constant when the separation $e$ varies at constant number of layers, $n_{0}$. The contribution to the force arising from the line energies can be evaluated as.

$$
2 \pi\left(\gamma+\gamma^{\prime}\right) \sum_{\imath=1}^{N_{\max }} \frac{1}{\tilde{r}_{\imath}} \cong \sqrt{2 N_{\max }+1} \pi \tilde{\lambda}\left(\frac{d}{R}\right)^{1 / 2} B
$$

According to the loop array model (see Section 3 ), $N_{\max }$ is given by the sample thickness $(\approx 1-2 \mathrm{~mm})$ at the outer boundary of the sphere-plane wedge shaped geometry, and is estimated to be around $10^{5}$ (the validity of the model in this region is discussed later). As a result, the contribution given by equation (21) to the net force is important. However, because $N_{\max }$ does not change significantly when the confined system is immersed in a reservoir (a crude calculation shows that $N_{\max }$ varies only by a few units when the surfaces are approached from a distance of a few micrometers down to contact: $\delta N_{\max } / N_{\max }<\delta e / R \approx 10^{-4}$ ), the force from equation (21) can be considered as a uniform repulsive background as illustrated by the dotted baselıne in Figure $3 \mathrm{~b}$ If we choose $B=10^{5} \mathrm{~J} / \mathrm{m}^{3}$ and $\tilde{\lambda}=2 \mathrm{~nm}$, two typical values expected for the experimental system studied here $[39,45]$, the background would amount to about $0.2 \mathrm{mN} / \mathrm{m}$ which is above the sensitivity of the surface force apparatus. Of course, the variation should be larger when the separation changes over a scale of the sphere radius; in this case $N_{\max }$ will decrease, vanishing when the sphere is so far away from the plane that the line 
tension contribution also vanishes. On the other hand, when the two surfaces contain only a droplet of liquid crystal not being surrounded by a reservoir, $N_{\max }$ grows continuously as the separation decreases. Accordingly, equation (21) shows an increasing repulsion as the contact is approached. This is consistant with the continous repulsion observed by Horn et al. at all separations for a thermotropic smectic droplet sandwiched between two mica surfaces [21].

The estimated value of $N_{\max }$ used to describe the experimental situation is actually not exact for two reasons. First, it was assumed that the Burgers vector, $b$, is constant over the entire varying thickness of the medium confined between the two walls. The measured force-distance profile indicates that this is indeed the case $(b=1)$ in the first few cells, $N_{\imath}$. Unfortunately, no experimental evidence can be gained for loops of higher order $(\imath>50)$. Actually the above assumption is questionable in view of the study carred out by Nallet and Prost for a similar confining geometry [39]. Indeed, it was shown that beyond a threshold thickness between a sphere and a flat, the Burgers vector of the confined lyotropic liquid crystal is no longer minimal but instead becomes a function of the position, $r$, of the loop and rises with increasing radius. In view of these results, the elastic free energy given by equation (5) becomes an explicit function of $\mathbf{b}$, and herse should be minimized with respect to both $b$ and $r$. Refining the model is a difficult task, as the experimental crossed-cylinder geometry is no longer equivalent to a sphere-plate geometry for large thicknesses. The loops of large radıus become curved and are no longer lying in planes. In addition the loops may even become screw-like as one moves along the dislocation, and they may also be intertwined, rarely remaining flat and pure edge-like. Note that Herke et al. have recently reported that the elastic stress of a thermotropic liquid crystal confined in a thick spherical wedge (a few microns) and under strain could be released by an avalanche of helical instabilities of screw dislocation lines [46]. Taking into account the occurrence of dislocations with large Burgers vectors would give a lower estimate for $N_{\max }$. Undoubtedly, the force contribution due to the line effects would also be lowered from the former overestimate. However, equation (21) suggests that even if this contribution to the net force is well above the sensitivity of the apparatus, it will not change or will not vary significantly over the separation range of the experimental observation, i.e., when about fifty dislocation loops are successively annihilated or created at the center. Consequently the line tensions affect the net force by generating a non-zero background. Although the oscillatory behaviour of the force profile expressed by the Derjaguin term is totally unchanged, the entire set of intersecting parabolae is translated upwards to a higher energy. Unfortunately there is no way to observe a constant background with the surface force apparatus technique. The procedure used to analyze the data yields no difference in the case of either a strictly zero or a finite constant force-distance profile, and hence we are unable to confirm the above prediction. A zero background has been used during the analysis of the experimental measurements by setting the force-distance profile at large separation to zero as illustrated in Figure 1a.

The first term, proportional to $\tilde{r}_{0}^{-1}$, in the summation is now considered. This term, which can be rewritten as $F_{0} / R=\pi \tilde{\lambda} B d \tilde{r}_{0}^{-1}$, is dominant in this series and can amount to relatively large magnitudes at the thicknesses at which a defect nucleates. This term is present only over surface separations for which the central stack is stretched, as shown in Figure $3 \mathrm{~b}$, and consequently exhibits a force-distance profile comprised of a set of discontinuous parts. The maximum extent of each section ranges from the critical thickness $e_{\mathrm{c}}$, where a new loop is nucleated, down to the separation where the stress has been totally released, i.e., at $e=n_{0} d$. As soon as the nucleation has occurred, the loop gains one order in the hierarchy of the array, becoming the loop $\imath=1$ with radius $r_{1}$. All the other existing loops, formerly of rank $\jmath$, also move by one order higher: $\imath=\jmath+1$. The summation term now includes the new loop $(\imath=1)$ together with all the loops $(\imath=\jmath+1)$ that existed prior to the nucleation; these loops adjust their radius accordingly to the new separation (a slight enlargement by about $\left(R d / 2 n_{\imath}\right)^{1 / 2}$ ) 
Thus the contribution to the net force of this additional loop is already integrated in the summation term analysed precedently. At the thicknesses where the nucleation occurs, the transition radii $\tilde{r}$ can be written as $\left(2 R \tilde{\lambda} e_{0}\right)^{1 / 3}$ according to equation (19). Using the same values as previously for $B$ and $\tilde{\lambda}$, a numerical application yields a radius $\tilde{r}$ of about $1 \mu \mathrm{m}$ for $n_{0}=2$ (the smallest radius possible) while $F_{0} / R$ is about $0.015 \mathrm{mN} / \mathrm{m}$ as displayed in Figure $3 \mathrm{~b}$. This force is just above the sensitivity of the apparatus. Thus, when the surfaces are brought closer together from the nucleation thickness, the radius of the loop, $r_{0}$, increases and the corresponding force decreases as $F_{0} / R \approx\left(e_{0}-e\right)^{-1 / 2}$ to first approximation. As a result the contribution to the net interaction diminishes rapidly, below the apparatus resolution. Accordingly, the symmetry of each parabola is broken relative to its minimum: the part of the parabola with a positive slope is affected, whereas to first approximation the contribution to the other part with a negative slope is negligible and well below the sensitivity of the apparatus (compare Figs. 3a and $3 b$ ). The equilibrium radii $\tilde{r}_{\imath}^{-1}$ were obtained numerically by successive minimizations of equation (5) using the same numerical values previously chosen for the parameters. The two effects that the line tensions have on the net force are displayed: the generation of a uniform background on which a discontinuous profile (analogous to a first-order transition) is superimposed.

It would be a great advantage to be able to record experimentally the force profile continuously, including the force regions of positive slope that correspond to the stretching of the central stack. From the dissymmetry of "parabolae", $\tilde{\lambda}$ could then be directly extracted, and $B$ would be obtained independently from the regular parabolic parts of the oscillations. Unfortunately, the distorded parts of the parabolae are not fully accessible with the surface force apparatus. Indeed their slopes are generally greater than the stiffness of the cantilever on which one mica surface is mounted, so that intrinsic mechanical instabilities occur.

To summarize, the elastic contribution of the interaction (Eq. (16)) is comprised of two terms of different magnitude, each arising from a different origin. The main term for which the Derjaguin approximation holds is due to the confinement of the stack of layers. It gives the oscillatory characteristics, amplitude and periodicity, to the force-distance profile. The second term arises from the line tensions of the edge dislocation loops. This gives slight corrections to the first contribution, by generating a non-zero constant background to the force and partly distorting the parabolic shape of every oscillation. These two deviations are not measurable with the surface forces apparatus and hence are of no consequence in the analysis of the data. The Derjaguin term is the only one relevant to analyze the oscillations of the force profile tracked by the surface forces apparatus (further discussed in Paragraph 4.3). On the other hand, the observed overall attractive background, which must have a non-elastic origin (see Paragraph 4.4), has little effect on the elastic wedge-shaped force because its variation is slight compared with the oscillation amplitudes.

4.2. Thermodynamic Equilibrium. - One particular concern in measurements dealing with mesophases is the achievement of thermodynamic equilibrium. A first aspect of this problem is the question of having the system under study at equilibrium before any force run is initiated. As recalled in Section 2, homeotropic alignment of the lamellar mesophase was obtained within a few hours, whereas longer times are often required for other systems. Also more than $24 \mathrm{~h}$ were allowed for equilibration (after immersion of the mica surfaces in the liquid crystal) before any force measurement. Secondly, in order to obtain valid information, it is crucial that the thermodynamic equilibrium is maintained throughout the force run. Creation or annihilation of edge dislocation involve structural rearrangements and energetic changes which are propagating from the nucleation site throughout the sample, and occur within transit times. In this study, the duration required to squeeze out or to incorporate a layer in the 
confined medium is of the order of a few seconds (note that this duration can last up to a few minutes for other systems).

The first fifteen oscillations, closest to the mica-mica contact, were tracked with both inward and outward movement of the surfaces, Figure $1 \mathrm{~b}$. During the outward movement, the surfaces jump out when the slope of the force exceeds the cantilever spring stiffness. The instability occurs after passing the minimum of the $n^{\text {th }}$ parabola during which the system incorporates a supplementary layer in the central stack by annihilation of the first dislocation loop. According to equation (12), the outward jump positions, hereafter called $l_{n}^{0}$, are expected to be very close to the locations of the minima, $l_{n}$, of each parabola, especially for the first ones. Indeed, using the experimental values of the spring constant, $K$, and the radius of curvature, $R$, of the surfaces, $K / R \approx 1.9 \times 10^{4} \mathrm{~N} / \mathrm{m}^{2}$, and $\Delta l_{n}^{0}=l_{n}^{0}-l_{n}$ is roughly $1 \%$ of the value of $l_{n}$ according to: $\left(l_{n}^{0}-l_{n}\right) / l_{n}=K /\left(2 \pi B R\right.$ ) (for example $\Delta l_{n}^{6} \approx 0.6 \mathrm{~nm}$ for the $6^{\text {th }}$ oscillation; Fig. 4 ). Note that at large separations, the slope of the force curve becomes smaller than $K$ and no more outward jumps are observed. This should occur at a surface separation $l_{n} \approx 265 \mathrm{~nm}$, which correlates well with the end of the observable discontinuities $(\approx 280 \mathrm{~nm})$. We recall that every minimum $l_{n}$ corresponds to a separation between the surfaces that is an integral multiple of $d$. Thus at thermodynamic equilibrium, the outward jump positions for the first oscillations must be a linear function of $n$, the order of the parabola. Taking into account the fact that the distance, $d^{\prime}$, between the mica boundaries and the nearest bilayer is not necessarily the same as that between two adjacent bilayers, $d$, the positions of the minima $l_{n}$ (or $l_{n}^{0}$ ) scale as $(n-2) d+2 d^{\prime}$. Figure 5 a demonstrates that this is the case. The slope of the least-squares fit to the data gives the reticular distance $d=8.8 \mathrm{~nm}$, which is exactly that measured for a bulk sample by X-ray scattering. The intercept, $d^{\prime}=6.2 \mathrm{~nm}$, is found to be slightly lower than $d$.

Similarly, the thermodynamic equilibrium condition can be checked using the positions $l_{n}^{\mathrm{i}}$ of the inward jumps. Note that the shift in distance due to the nucleation occurrence (towards $e_{0}$ ) is very small. Indeed, when the line effects are taken into account, this shift, given by $\epsilon$ (Eq. (18)), ranges from 0.04 to $0.3 \mathrm{~nm}$ for the first fifteen oscillations considered, which is below, or at most equal to, the distance resolution of the surface forces apparatus. Thus, since $l_{n}^{1}$ is almost equal to $e_{0}$, at thermodynamic equilibrium, $l_{n}^{1}$ must scale as $((n-1) n)^{1 / 2} d$, as shown in Figure 5b. Again the extracted slope from the best linear fit is $8.8 \mathrm{~nm}$. As before, and for the same reason, the data do not extrapolate to the origin. Furthermore, the first two points lie slightly away from the fit line. This is because the compression modulus of the two nearest surface layers departs from the bulk modulus (see also Paragraph 4.3).

In consideration that the values of $l_{n}^{1}$, at small separations, have been obtained at thermodynamic equlibrium, the activation barrier $\Delta E_{0}\left(r_{\mathrm{c}}\right)$ given by equation (20) is now discussed. The height of the barrier does not seem to present any large hindrance for the thermal nucleation of dislocation loops at these separations. According to Pershan and Prost [36], the nucleation rate per unit volume has the following expression,

$$
f \cong f_{0} \exp \left(-\Delta E\left(r_{\mathrm{c}}\right) / k_{\mathrm{B}} T\right)
$$

where $f_{0}$ is a characteristic rate involving some microscopic processes (typically, the nucleation rate of a thermally activated pore with a molecular length radius). Following their procedure to estimate $f_{0}$ in the case of a thermotropic smectic, a similar value is found for a lyotropic smectic, namely $f_{0} \cong 10^{33} \mathrm{~s}^{-1} \mathrm{~cm}^{-3}$. The threshold rate, $f_{L}$, can be estimated as follows. The inward jump is experimentally induced when the walls are at the critical separation, $e_{c}$ (see Section 3), i.e., as soon as the edge dislocation loop is activated in the central cell (volume $\approx n_{0} d \pi \rho_{1}^{2}$ with a lateral extension given by $\rho_{1} \approx(R d)^{1 / 2}$ ). This implies that $f_{L}$ actually scales as $n_{0}^{-1}$. As an illustration, in the case of a central cell comprised of $n_{0}=15$ layers, and knowing that the mean time between two successive measurements during the force run is about a few 


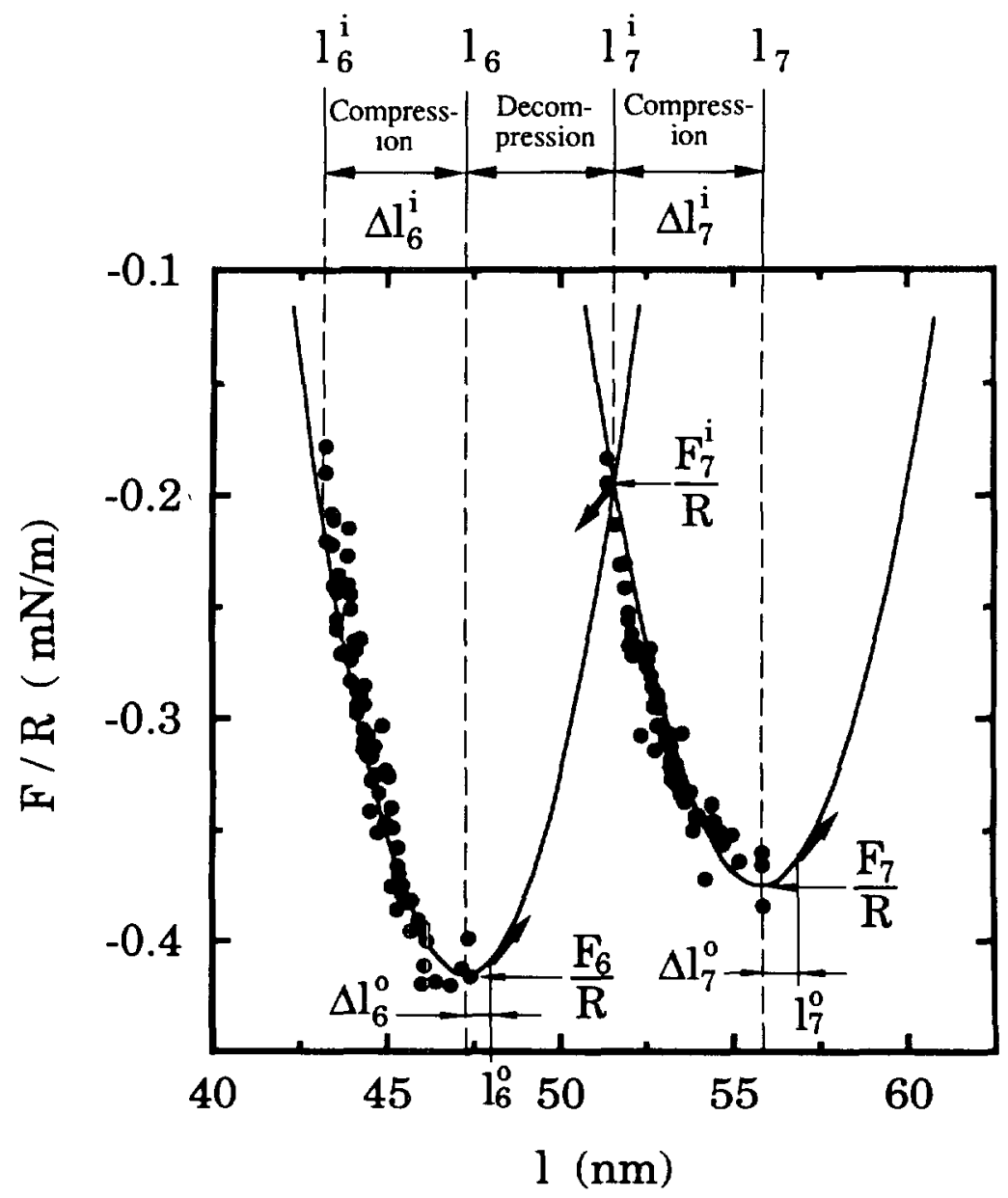

Fig 4 - An expansion of all the data shown in Figure $1 \mathrm{~b}$ for the $6^{\text {th }}$ and $7^{\text {th }}$ oscillations. The solid lines are parabolic fits using $B=1.8 \times 10^{5} \mathrm{~J} / \mathrm{m}^{3}$ in equation (12) The large arrows show the theoretical jump positions. The inward jump positions $l_{n}^{1}$ are observed to be located at the intersection of the two adjacent parabolae as required for measurements carried out at the thermodynamic equilibrium. The outward jump positions $l_{n}^{0}$ are very close to the bottom of the parabola $l_{n}$. The theoretical difference $\Delta l_{n}^{0}=l_{n}^{0}-l_{n}$ is roughly $1 \%$ of the value of $l_{n}$; for instance, $\Delta l_{6}^{0} \approx 5 \AA$ and $\Delta l_{7}^{0} \approx 6 \AA$

seconds, we can estimate $f_{L} \cong 10^{9} \mathrm{~s}^{-1} \mathrm{~cm}^{-3}$. The corresponding limit for the energy barrier would be $\Delta E_{L}\left(r_{\mathrm{c}}\right) \cong 55 k T$. Substitution of this value into equations (18) and (20) yields the estimate of an upper bound $\tilde{\lambda}$, which appears to be smaller than $0.3 \mathrm{~nm}$, a surprisingly low value. If one allows the occurrence of the nucleation to be delayed, application of a further larger strain would diminish the height of the barrier accordingly. A delay of $e_{c}-e=0.3 \mathrm{~nm}$ is a reasonable limit and still within the distance resolution of the surface forces apparatus, as well as the accuracy in the measurements of the positions of the inward jumps, $l_{n}^{\mathrm{i}}$. For this upper bound, $\tilde{\lambda}$ could increase up to $0.8 \mathrm{~nm}$. Using the measured compressibility modulus, $B$, of the stack of the layers extracted independently from the data (see Paragraph 4.3), the penetration length $\lambda=\left(\kappa_{\mathrm{c}} / d B\right)^{1 / 2}$ is expected to be about $1.6 \mathrm{~nm}$ when the membrane bending rigidity 

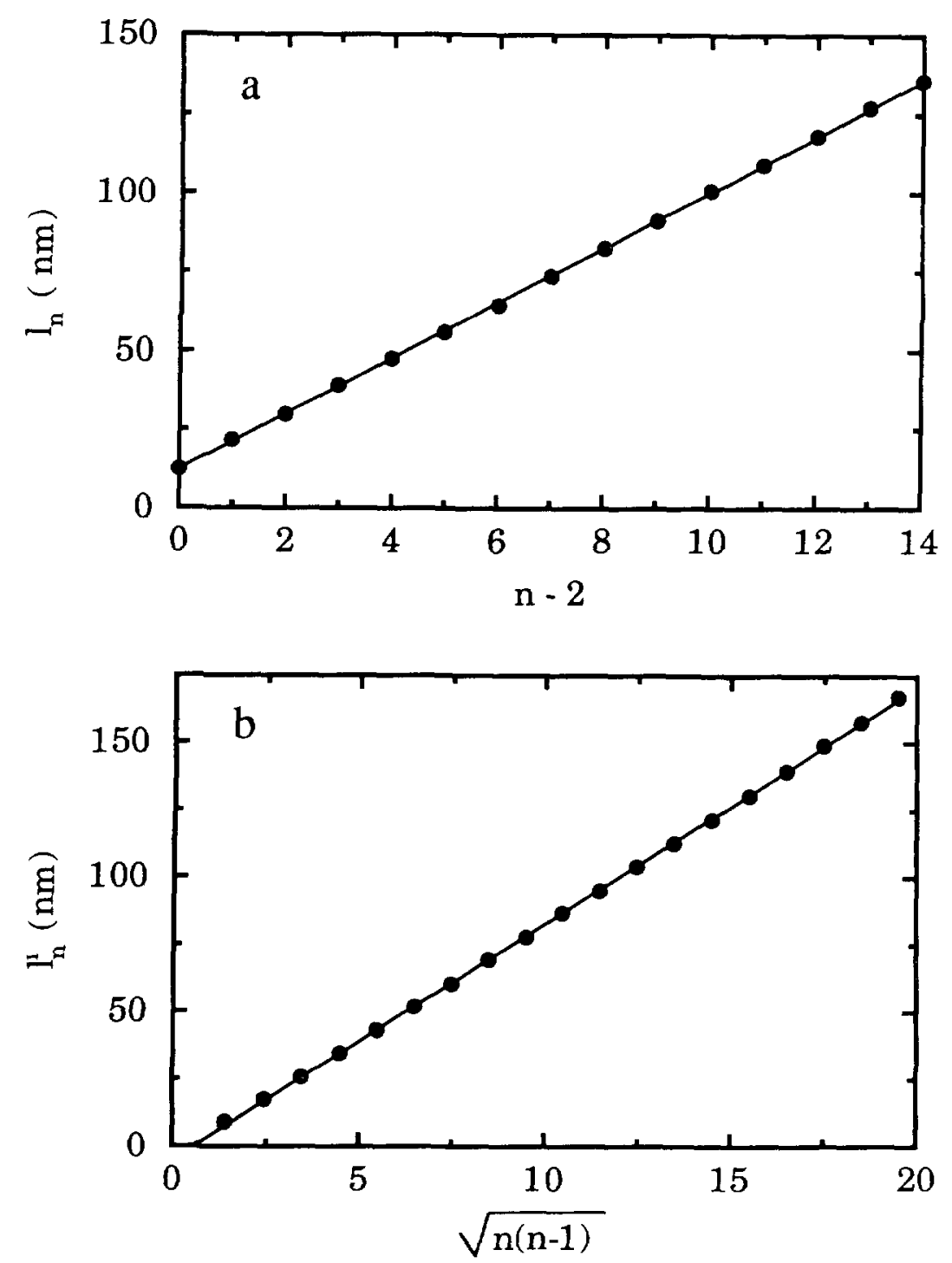

Fig 5. - For each oscillation $n$, the positions of the minimum $l_{n}$ are plotted against the rank $n-2$ (a), and those of the inward jump $l_{n}^{2}$ against $\sqrt{n(n-1)}$ (b). The solid lines are the least-squares fits to the data giving a reticular distance of $88 \mathrm{~nm}$ in agreement with the X-ray measurement. The fact that the measured values are so close to the fitted line indicates that the forces were measured at the thermodynamic equilibruum

is assumed to be $\kappa_{\mathrm{c}}=k_{\mathrm{B}} T$. This is still twice as large as the upper bound estimated above, since $\tilde{\lambda}$ is expected to be equal to $\lambda$ at least. Even if one questions the actual value taken for $\kappa_{\mathrm{c}}$ (according to Refs. [48] and [49], the electrostatic contribution alone would account for $\approx 0.7 k_{\mathrm{B}} T$, leading to a penetration length of $1.3 \mathrm{~nm}$ ), our model seems to overestimate the line tension effects (note this has no implications in the data analysis) Some reasons can be invoked. For instance, it may be questionable to use an expression for the far-field energy derived for a dislocation loop trapped between two parallel plates where layers far from the 
loop have zero external stress. In the experimental situation, it is possible that the inside and the outside of the parabola of strain become less well differentiated, especially when walls are close to each other. As pointed out by de Gennes [49], some of the defects may bear little resemblance to dislocations in a bulk sample. as the core region may be spread along the plane of the layers to such an extent that the concept of a dislocation line is no longer applicable. As a result, it may be energetically less costly to have continuously distorted layers contouring the curved walls. Whatever the cause, the above discussion emphasizes that some systems with large penetration length could give rise to a very erratic force-distance profile. This was observed for many systems we studied [50], and was not always due to difficulties in achieving a homeotropic alignement along the surfaces. For these systems, the approach of the two walls towards contact resulted in a force-distance profile comprised of repulsive oscillations, but with inward jumps with no regular positions, i.e., with no direct relation with a constant integer multiple of the reticular distance. The history of the sample under study is now of great importance; the force-distance profile will depend on the successive compression/dilation cycles carried out previously. Thus, it is observed that either the oscillations may be different, or the magnitude of the same oscillations may show a great variability. We think that the force profiles presented by Abillon and Perez are a good lllustration of these characteristics where marked non-equilibrium effects are present, as recognized by the authors [25]. Such a behavior might be interpreted in terms of too high an energy barrier to nucleate the dislocations, so that the nucleation of an edge dislocation loop is delayed. To overcome the barrier, large strains exceeding a few layer thicknesses must be applied before the stress is released by the occurrence of a more likely nucleation. Thus the compressive force will follow a path located well above the supposed set of intersecting parabolae. On compression, the force-distance profile will deviate rapidly from the pure elastic parabolic shape (Eqs. (12) and (16); Fig. 4). On dilation, another path will be followed, as the annihilation of a dislocation is not equivalent to its creation.

4.3. Compressional Elastic Modulus. - After having checked that the experiment has been performed at the thermodynamıc equilibrıum, equation (12) is used to fit the oscillations of the force profile in order to extract the compressibllity modulus $B$. Figure 4 shows an enlargement of the force around the sixth and the seventh oscillations from contact. The full circles are the experimental data whlle the solid lines are parabolic fits using $B=1.8 \times 10^{5} \mathrm{~J} / \mathrm{m}^{3}$. Because of the contribution of the long-range attractive background, the fit is applied onto the difference $\left(F-F_{n}\right) / R$, where $F_{n}$ is the non-zero force at the minima $l_{n}$ The non-dependence of $B$ upon $n$ is checked by plotting $\left(F-F_{n}\right) l_{n} / \pi R=B \Delta l_{n}^{2}$ for $n=5,6$ and 12 (Fig. 6). These points lie about a straight line of slope $B=1.7 \times 10^{5} \mathrm{~J} / \mathrm{m}^{3}$ A more refined analysis takes into account the decrease in $d$ for the first two layers, as the distance between the first bilayer and the mica surface is $d^{\prime}=6.2 \mathrm{~nm}$. This gives a correction $\alpha_{n}$ of the equation above, vanishing as $\mathrm{n}$ increases: $\alpha_{n}=3 l_{n}^{0} /\left(3(n-2) d+4 d^{\prime}\right)$ (the compressional modulus for the first two layers in the vicinity of the mica walls is estimated to be $\approx 1.5 B$ using the theoretical expression (Eq. (24)) for layers stabilized by electrostatic forces; see below) With the correction, for the first 15 oscillations we found: $B=(1.9 \pm 0.4) \times 10^{5} \mathrm{~J} / \mathrm{m}^{3}$ in close agreement with that inferred from high-resolution X-ray scattering in the same system [45]. Note that $B$ would be independent of the number of layers $n$ if the smectic phase were a perfect $1 \mathrm{D}$ crystal, but for a quasl-ordered system the theory predicts a Landau-Peierls type correction $B_{n}=B\left(1+A \operatorname{Ln}\left(l_{n} / d\right)\right)^{4 / 5}[51]$. The same theory indicates a correction of only a few percent when the thickness $l_{n}$ is more than one thousand layers. Our measurements on 2 to 16 oscillations show that this effect is negligible.

A direct comparison with the theory is also possible. The modulus of layer compression $B$, at constant chemical potential, reflects the nature of the interaction between two membranes 


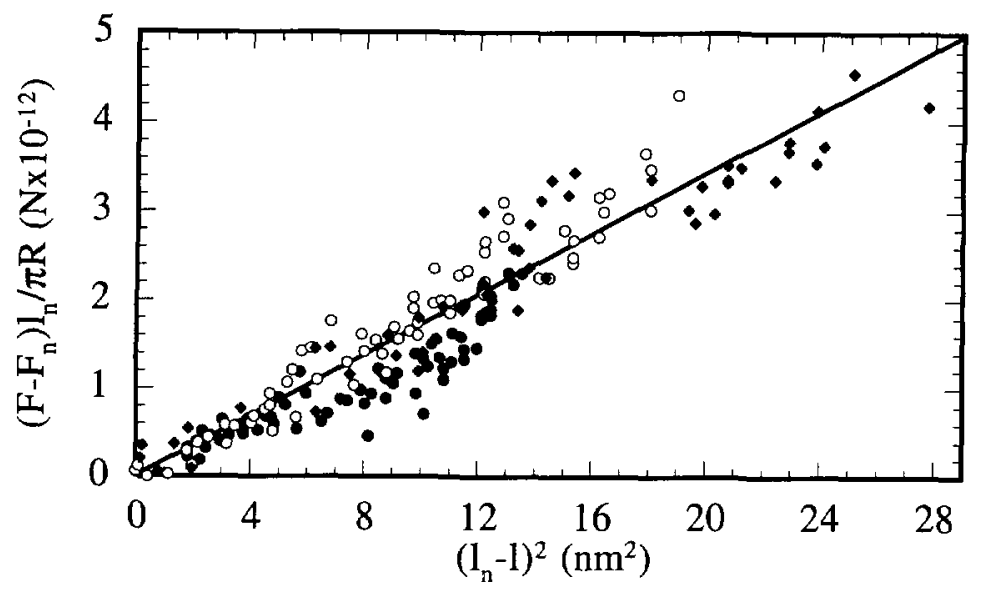

Fig. 6. - The data from the $5^{\text {th }}(\bullet), 6^{\text {th }}(0)$, and $12^{\text {th }}(\bullet)$ oscillations are plotted according to $\left(F_{n}^{1}-F_{n}\right) l_{n} / p R=B \Delta l_{n}^{2}$, where $\left(F_{n}^{1}-F_{n}\right) / 2 \pi R$ is the total energy expanded on going from $l_{n}$ to $l_{n}^{1}$ (see Fig. 4) and $\Delta l_{n}^{1}=l_{n}^{1}-l_{n}$. The straight line fits to these points gives $B=1.7 \pm 02 \times 10^{5} \mathrm{~J} / \mathrm{m}^{3}$ The obtained layer compressibility modulus $B$ is remarkably close to that derived theoretically (Eq. (24)).

in the stack:

$$
B=d\left(\partial^{2} E / \partial d^{2}\right)_{n}
$$

where $E$ is the free energy per unit surface area and $n$ is the number of layers per unit length $(n=1 / d)$. For the water-swollen system studied here, the interactions between the lamellae have been shown to be purely electrostatic [45]. The electrostatic pressure exerted between two membranes has been derived by Cowley et al. [52]. Using equation (23) for a system with a high density of charges and large reticular distance, $B$ reads:

$$
B=\frac{\pi^{2} k_{\mathrm{B}} T d}{2 L(d-\delta)^{3}}\left[1-3 \frac{\Sigma}{a L(d-\delta)}+6 \frac{\Sigma^{2}}{\alpha^{2} L^{2}(d-\delta)^{2}}+\ldots\right]
$$

where $\delta$ is the bilayer thickness $(2.0 \mathrm{~nm},[45]), \alpha$ is the dissociation coefficient of counterions (assumed to be unity as there is about one SDS polar head for 7-8 pentanol molecules at each bilayer interface), $\Sigma$ is the surface area per charged polar head ( $\Sigma \approx 2 \mathrm{~nm}^{2}$, deduced from the sample composition), and $L=\pi e^{2} / \epsilon k T$ is a characteristic length for an aqueous solution ( $L=2.2 \mathrm{~nm}$ at room temperature). With these parameters we obtain $B=1.82 \times 10^{5} \mathrm{~J} / \mathrm{m}^{3}$, in remarkable agreement with the measured value.

4.4. Confinement EFfects. - The existence of the background attraction with such a long-range may appear quite surprising. Such a feature has also been observed in all the systems studied so far $[23,24]$ and may reach a large magnitude, as was reported in an oilswollen lamellar phase most-likely stabilized by an undulation interaction [53]. The behavior therefore looks quite general for a stack of layers confined between walls. First, let us point out that this attraction cannot be accounted for by dispersion forces, even if one carries out the full calculation of the interaction between bodies across multilayer systems [54]. Thus, the generation of this long-range attractive background must originate from different causes.

The first possibility would be a Casimir-like effect where some orientational fluctuations of the structured phase are suppressed by confinement. This was recently analyzed by Ajdari $e t$ 
al. who showed that long-range forces may arise from the boundary conditions imposed by two rigid walls on the director fluctuations in nematics [30,31]. They extended their results for smectic and columnar phases in different confining geometries. The basic idea is to consider that the liquid crystal fluctuates with decoupled modes of well-defined amplitude (corresponding to an energy $\approx k_{\mathrm{B}} T / 2$ ). Thus the fluctuating medium can be seen as a collection of ideal modes or particles with a characteristic size related to their wavelength. In a confining geometry with symmetric boundary conditions, the modes with a size larger than the wall-separation are excluded. Accordingly, the corresponding osmotic pressure in the gap is lowered with a decreasing separation and the resulting force is an attraction. This is similar to the attractive entropic depletion in colloidal suspensions where the particle density is lowered in the gap [55]. For a smectic slab of thickness $e$, in a homeotropic alignment between two parallel plates, the energy density per unit area is [56]:

$$
f=-\frac{\zeta(2)}{16 \pi} \frac{k_{\mathrm{B}} T}{\lambda e}
$$

where $\zeta$ is the Riemann zeta function and $\lambda$ is the penetration length. Note that this interaction is longer range than the van der Waals attraction. The magnitude of the effect is difficult to estimate, as use of equation (25), derived for a planar geometry, may not be directly applicable in the crossed-cylinder geometry of the experiment. If the Derjaguin approximation ( $F=$ $2 \pi R f$ ) is crudely used [44], the magnitude of the effect appears to be roughly a little more than one order of magnitude too low (keeping the same expected penetration length, $\lambda \approx$ $1.6 \mathrm{~nm})$. However, the Derjaguin approximation is not expected to hold, as the layers are both compressed and stretched between two adjacent dislocations. A more refined analysis is required. Unfortunately, an analytical derivation seems quite untractable, and possibly, only a numerical analysis might give some insights into the correct order of magnitude of the effect.

A second possibility can be invoked to account for the long-range attractive background. It is closely related to the first idea, but here fluctuations over smaller scales are considered. The attraction is the consequence of the interaction between the walls and the structured sample. The order parameter of the structured medium is affected by the presence of each wall over a finite region located in their vicinity. The stronger the anchoring, the larger the enhancement of the order parameter within this adjacent region. As first suggested by Marčelja and Radić [29], and confirmed later by others [57-59] for a nematic liquid crystal, with symmetric boundaries, the surface excess free energy decreases when the separation between the walls is reduced as soon as the two adjacent layers overlap. Consequently the two walls attract each other. An analogous approach can be followed for a smectic confined between two parallel plates a distance $e$ apart. Following Sheng $[57,58]$, the excess free energy per unit area in a Landau expansion to the lowest orders is:

$$
\Delta f=\int_{-e / 2}^{e / 2}\left[\frac{r}{2} \eta^{2}+\frac{c}{2}(\nabla \eta)^{2}\right] \mathrm{d} z+\int_{-e / 2}^{e / 2} V_{s}[\delta(z-e / 2)+\delta(z-e / 2)] \mathrm{d} z
$$

where at the distance $z$ from one wall, $\eta(z)=\rho(z)-\rho_{\mathrm{b}}$ is the excess order parameter relative to the bulk order parameter, $\rho_{\mathrm{b}}$; the amplitude of the density modulation is $\rho(z) ; c$ and $r$ are two positive coefficients, while $V_{\mathbf{s}}(r)$ is a surface potential due to the walls. To simplify, $V_{\mathrm{s}}(r)$ is considered to be only a contact potential of zero range (the same conclusions would be reached provided that this potential range is much lower than the smectic coherence length $\xi$ ). As the walls are supposed to induce some enhancement of the order in their vicinity, the first derivative of the surface potential $V_{\mathbf{s}}\left(\eta((z))\right.$ is negative: $V_{\mathrm{s}}^{\prime}=\mathrm{d} V_{\mathbf{s}} / \mathrm{d} \rho<0$. The Euler-Lagrange 
equation requires:

$$
\xi^{2} \frac{\mathrm{d}^{2} \eta}{\mathrm{d} z^{2}}=\eta
$$

together with the boundary conditions,

$$
c\left(\frac{\mathrm{d} \eta}{\mathrm{d} z}\right)_{z=-e / 2}=V_{\mathrm{s}}^{\prime} \text { and } c\left(\frac{\mathrm{d} \eta}{\mathrm{d} z}\right)_{z=e / 2}=-V_{\mathrm{s}}^{\prime},
$$

where $\xi=(c / r)^{1 / 2}$. The solution satisfying the boundary conditions is:

$$
\eta(z)=\frac{\xi V_{s}^{\prime}}{c} \frac{\cosh (z / \xi)}{\sinh (e / 2 \xi)}
$$

The disjoining pressure, $\pi_{\mathrm{D}}=\mathrm{d} \Delta F / \mathrm{d} e$, i.e., the force per unit area required to hold the plates at a distance $e$ apart is then:

$$
\pi_{\mathrm{D}}=\frac{V_{\mathrm{s}}^{\prime 2}}{r \xi} \operatorname{coth}(e / 2 \xi)
$$

At large separations, $e>2 \xi$, the plates attract each other exponentially with a decay length $\xi$. As noted by others $[29,57,58,60]$ the exponential behaviour founded here is a characteristic of the contact short-range potential assumed for the surface forces. The interaction range is given by $\xi$ which is closely related to the correlation length of the order parameter.

Quantitative comparison with experiment is a hard task, because the phenomenological coefficients $c$ and $r$ are unknown. On the other hand, the correlation length $\xi$ is expected to be of the order of a few layers. But at the approach of a weakly first-order or second-order phase transition the correlation length diverges, following a power law. Therefore, force studies for systems in the vicinity of such phase transitions would give clues about the nature of the attractive background, and whether it arises from the modified order parameter near the walls. The answer looks positive in view of the results obtained with lyotropic systems exhibiting either a second-order/weakly first-order phase transition between a nematic and a lamellar phase [26] or a first-order transition between a sponge and a lamellar phase [27].

Before concluding, we would like to underline the increase of the background at short separations, i.e., $e<40 \mathrm{~nm}$ (Fig. 1b). As we have previously noticed, interactions between the dislocation loops and the surfaces are expected when the thickness of the sample is the order of the penetration length [41]. Since the interactions are repulsive, an extra repulsion between the walls must develop for separations of a few layers as observed in the data.

\section{Conclusions}

The forces measured at thermodynamic equilibrium between two solid surfaces separated by a film of lamellar phase in a homeotropic alignment present two kinds of specific behavior that arise from two different origins. The first contribution, which is undoubtedly identified, is of elastic nature It generates a force-distance profile comprised of a set of intersecting parabolae. The minima of these parabolae are located on a long-range attractive background, which is induced by another contribution. We attribute its origin to some effects of entropic nature.

At small separations, the geometry of the confinement is equivalent to a sphere against a plane. In order to fill the confined space of continuously varying thickness in a homeotropic alignment, the layered system generates an array of edge dislocation loops. Thus the elastic response of the system to any strain applied normal to the walls separates into two contributions. The first one arises from the stress experienced by the central stack, while the second 
one results from the line tensions of the edge dislocations. We have shown that the latter leads only to a weak perturbation of the measurements. Beyond a threshold strain (about one half layer) which is virtually independent of the number of layers in the central stack, the elastic stress is relaxed when a layer is added to or expelled from the stack. This is achieved via a dynamical process. Under a compression or a decompression, a loop is nucleated or annihilated, respectively, at the center of the confinement. Between two dynamical events, the interaction between the surfaces is directly related, by the Derjaguin equivalence, to the elastic stress experienced by the central stack. The corresponding force has the shape of a parabola centered at each thickness which is an integral multiple of the layer thickness so that the stress in the central stack is nil. Thus the entire force profile is made of a set of intersecting parabolae of constant period between the minima, equal to the reticular distance for the reported system. From every parabola the layer compressibility modulus is directly extracted with accuracy. The measured value is in very good agreement with the theory and other experiments performed on the same system.

Two additional effects take place in a structured system confined between two rigid walls. Both arise from restrictions in the fluctuations of the layers. As the space is limited in a confined geometry, several modes of the orientational fluctuations are forbidden. The number of prohibited modes increases with a decreasing separation, leading to a long-range attraction between the walls. Moreover, in the vicinity of walls, the order parameter of the mesophase is modified and enhanced if the surfaces induce ordering. As previously when the surface separation is reduced, the overlapping of the adjacent layers with enhanced structure leads to an attraction between the walls, which may become of long-range near a phase transition. We attempted to compare these two effects with the experimentally observed long-range attractive background on which the set of parabolae is superimposed. We failed to quantify correctly each of these two effects, consequently the origin of the background is still not fully clear.

However, in order to identify which of the two contributions is dominant, it would be interesting to affect their range. The approach towards a phase transition would increase the correlation length of the order parameter, and accordingly the range of the related structural interaction Such experiments have been already started and have suggested that the attractive background originates from a modification of the order parameter induced by the walls [26,27]. In a similar manner, the other contribution may be tested in tuning the penetration length. To this end, the concentration of the co-surfactant or/and the length of their aliphatic chains can be changed. This second study would be also of great interest in regard to the activation barrier of the defect nucleation. Many experiments by their irregular results have suggested that the barrier is often larger than many $k_{\mathrm{B}} T$. Affecting the height of the activation barrier could be performed by tuning down the penetration length.

In conclusion, if our study gives a satisfactory description and understanding of the interactions of elastic nature, some complementary investigations are still called up, in particular those of entropic nature.

\section{Acknowledgments}

Experimental parts of this work were carried out at the Department of Applied Mathematics, Canberra, Australia. We thank B.W. Ninham for his hospitality and use of the laboratory. Discussions with D. Roux, J. Prost, A. Ajdari and B.W. Ninham are gratefully acknowledged. 


\section{Appendix A}

In this Appendix, we give a more general method to calculate directly the net elastic force between a sphere and a plate immersed in a smectic mesophase. An array of $N_{\max }$ concentric dislocation loops is still retained to keep a homeotropic alignment between the surfaces. Keeping the same definitions as those in the main text, recall that the whole elastic energy is the sum of the elastic energies $E_{\imath}\left(e, r_{\imath}\right)$ in each cell (independent):

$$
E_{T}(e)=\sum_{\imath=0}^{N_{\max }} E_{\imath}\left(e, r_{\imath}\right)
$$

The net elastic energy, $E_{\imath}$, stored in each cell $\imath$ is the sum of three contributions as discussed previously:

$$
E_{\imath}=E_{\mathrm{W}_{\imath}}+E_{\mathrm{F}_{\imath}}+E_{\mathrm{C}_{\imath}}
$$

The first one is the wedge confinement energy, $E_{W_{1}}$ :

$$
E_{\mathrm{W}_{\imath}}\left(e, r_{\imath}\right)=\int_{\rho_{\imath}}^{r_{\imath}} 2 \pi r \mathrm{~d} r f\left(h(r), n_{\imath}\right)+\int_{r_{\imath}}^{\rho_{\imath+1}} 2 \pi r \mathrm{~d} r f\left(h(r), n_{\imath}+1\right)
$$

where $f(h, n)$ is the elastic free energy density of $n$ parallel smectic layers constrained over a thickness $h$. The second and the third ones are the line contributions as defined in the main text:

$$
\begin{aligned}
& E_{\mathrm{F}_{2}}\left(r_{2}\right)=2 \pi r_{2} \gamma \\
& E_{\mathrm{C}_{2}}\left(r_{2}\right)=2 \pi r_{2} \gamma^{\prime}
\end{aligned}
$$

Minimizing (A.2) with respect to $r_{\imath}$ gives the equilibrium radii $\tilde{r}_{\imath}$ of the dislocation loops:

$$
\begin{gathered}
\partial E_{\imath} / \partial \tilde{r}_{\imath}=0 \\
2 \pi \tilde{r}_{\imath}\left(f\left(h\left(\tilde{r}_{\imath}\right), n_{\imath}\right)-f\left(h\left(\tilde{r}_{\imath}\right), n_{\imath}+1\right)\right)+2 \pi\left(\gamma+\gamma^{\prime}\right)=0
\end{gathered}
$$

The net interaction, $F_{T}$, between the plane and the sphere is:

$$
F_{T}(e)=-\frac{\mathrm{d} E_{T}}{\mathrm{~d} e}
$$

The force can be rewritten as:

$$
F_{T}(e)=-\sum_{\imath=0}^{N_{\max }} \frac{\partial E_{\imath}}{\partial e}+\frac{\partial E_{\imath}}{\partial r_{\imath}} \frac{\mathrm{d} r_{\imath}}{\mathrm{d} e}
$$

The Euler-Lagrange condition imposes $\partial E_{\imath} / \partial r_{\imath}=0$, hence

$$
F_{T}(e)=-\sum_{\imath=0}^{N_{\max }} \frac{\partial E_{\imath}}{\partial e}
$$

According to (A.3) and (A.4), only the wedge contribution has an explicit dependence on $e$. Hence,

$$
F_{T}(e)=-\sum_{i=0}^{N_{\max }} \frac{\partial E_{\mathrm{W}_{i}}}{\partial e}
$$


For each cell, we obtain

$$
\frac{\partial E_{\mathrm{W}_{\imath}}}{\partial e}=\int_{\rho_{\mathrm{l}}}^{\bar{r}_{\imath}} 2 \pi r \mathrm{~d} r f^{\prime}\left(e+r^{2} / 2 R, n_{\imath}\right)+\int_{\bar{r}_{\mathrm{t}}}^{\rho_{\imath+1}} 2 \pi r \mathrm{~d} r f^{\prime}\left(e+r^{2} / 2 R, n_{\imath}+1\right)
$$

where $f^{\prime}$ is the first derivative of $f$ with respect to $e$. According to the sphere-plane geometry, this derivative can be rewritten as $\frac{R}{r} \frac{\partial f}{\partial r}$, and since by definition $h\left(\rho_{\imath}\right)=n_{\imath} d$, the elastic stress is nil at the cell boundaries provided that $i>0$, and therefore the elastic force in each of these cells $F_{2}$ is:

$$
-\frac{\partial E_{\mathrm{W}_{\mathrm{t}}}}{\partial e}=2 \pi R\left(f\left(e+\tilde{r}_{\imath}^{2} / 2 R, n_{1}+1\right)+f\left(e+\tilde{r}_{\imath}^{2} / 2 R, n_{\imath}\right)\right)
$$

Alternatively, and from the Euler-Lagrange equation (A5), the elastic forces may be written:

$$
F_{\imath}=2 \pi R\left(\gamma+\gamma^{\prime}\right) / \tilde{r}_{\imath}
$$

Note that if the line tensions are neglected $\left(\gamma+\gamma^{\prime}=0\right)$ the cells $\imath>0$ do not contribute to the net elastic force.

Now one has to consider the central cell which is special since the thickness at its inner boundary $(r=0)$ is $e$ (usually a non-integer multiple of the layer thickness $d$ ). Accordingly, the supplementary term arising from (A10) for $\imath=0$ is

$$
F_{0}=2 \pi R f\left(e, n_{0}\right)
$$

With the following elastic free energy density, where $B$ is the layer compressibility modulus,

$$
f(h, n)=\frac{B}{2} \frac{(h-n d)^{2}}{n d}
$$

we obtain the net elastic force between the sphere and the plane

$$
F_{T}(e)=\pi R B \frac{\left(e-n_{0} d\right)^{2}}{n_{0} d}+2 \pi R\left(\gamma+\gamma^{\prime}\right) \sum_{0}^{N_{\max }} \frac{1}{\tilde{r}_{2}}
$$

\section{References}

[1] Israelachvili J N., Intermolecular and Surface Forces (Academic Press, London, 1992).

[2] Hunter R.J., Foundations of Colloid Science, Vol. 1 (Clarendon Press, Oxford, 1987) p. 673.

[3] Derjaguin B.V. and Abrikosova I.I., Disc. Faraday Soc. 18 (1954) 33.

[4] Derjaguin B.V., Abrikosova I.I. and Lifshitz E M., Q. Rev. 10 (1956) 295.

[5] Black W., de Jongh J.G. V, Overbeek J.T.G. and Sparnaay M.J, Trans. Faraday Soc. 56 (1960) 1597.

[6] Tabor D. and Winterton R.H.S., Proc. Roy. Soc. A 312 (1969) 435.

[7] Israelachvili J.N. and Tabor D., Proc. $R$ Soc. Lond A 331 (1972) 19.

[8] Israelachvili J. N. and Adams G.E., J. Chem. Soc., Faraday Trans. I 74 (1978) 975.

[9] Pashley R.M., J Collond Interface Scı. 83 (1981) 531.

[10] Shubin V.E. and Kékicheff P., J Collond Interface Scr. 155 (1993) 108. 
[11] Kékicheff P., Marčelja S., Senden T.J. and Shubin V.E , J. Chem. Phys. 99 (1993) 6098.

[12] Klein J., Nature 288 (1980) 248; Adv. Collond Interface Scq. 16 (1982) 101; J. Collond Interface Scr. 111 (1986) 305

[13] Israelachvilı J.N., Tirell M., Klein J and Almog Y., Macromolec. 17 (1984) 204.

[14] Evans E. and Needham D, Macromolec. 21 (1988) 1822

[15] Richetti P. and Kékicheff P, Phys. Rev. Lett. 68 (1992) 1951.

[16] Kékıcheff P, Nallet F. and Richetti P., J. Phys. II France 4 (1994) 735.

[17] Horn R.G and Israelachvili J N., J Chem. Phys. 75 (1981) 1400.

[18] Christenson H.K., J Chem. Phys. 78 (1983) 6906.

[19] Attard P. and Parker J.L, J. Phys. Chem. 96 (1992) 5086.

[20] Parker J.L., Richetti P, Kékicheff P. and Sarman S., Phys. Rev. Lett. 68 (1992) 1955.

[21] Horn R.G., Israelachvili J.N. and Perez E., J. Phys. (Paris) 42 (1981) 39.

[22] Ter-Minassian-Saraga L and Perez E., Collonds Surf 12 (1984) 213.

[23] Kékicheff P. and Christenson H.K., Phys Rev Lett. 63 (1989) 2823

[24] Richetti P., Kékicheff P., Parker J.L. and Ninham B.W., Nature 346 (1990) 252.

[25] Abillon O. and Perez E, J. Phys. France 51 (1990) 2543.

[26] Moreau L., Richettı P. and Baross P, Phys. Rev Lett. 73 (1994) 3556.

[27] Antelmı D.A., Kékicheff $P$ and Richetti P., J. Phys. II France 5 (1994) 103.

[28] de Gennes P.-G and Prost J., The Physics of Liquid Crystals (Clarendon Press, Oxford, 1993).

[29] Marčelja S. and Radić N., Chem. Phys. Lett. 42 (1976) 129.

[30] Ajdarı A, Pelitı L and Prost J., Phys Rev. Lett. 66 (1991) 1481

[31] Ajdarı A, Duplantıer B., Hone D., Pelıtı L and Prost J., J. Phys. II France 2 (1992) 487.

[32] Kékicheff P., Grabielle-Madelmont C. and Ollivon M., J. Collond Interface Scı. 131 (1989) 112.

[33] Parker J. L., Christenson H.K. and Ninham B.W., Rev. Scı. Instrum. 60 (1989) 3135.

[34] Israelachvili J.N., J Collond Interface Sci. 44 (1973) 259.

[35] Marra J. and Hair M L., J Collond Interface Sci. 128 (1989) 511

[36] Pershan P.S. and Prost J., J. Appl. Phys 46 (1975) 2343.

[37] Meyer R.B., Stebler B and Lagerwall S.T., Phys Rev Lett 41 (1978) 1393.

[38] Chan W K. and Webb W W., J. Phys. (Parrs) 42 (1981) 1007.

[39] Nallet F and Prost J, Europhys. Lett. 4 (1987) 307.

[40] de Gennes P.-G., C.R. Hebd. Séan Acad. Scı. B 275 (1972) 549.

[41] Pershan P S., J. Appl Phys. 45 (1974) 1590.

[42] Kléman M., Points, Lınes and Walls: in Liquid Crystals, Magnetıc Systems, and Various Ordered Media (Wiley, New York, 1983).

[43] Lejcek L. and Oswald P., J. Phys. II France 1 (1991) 931.

[44] Derjaguin B.V., Kollond $Z 69$ (1934) 155.

[45] Roux D. and Safinya C.R., J. Phys. France 49 (1988) 307.

[46] Herke R A., Clark N.A. and Handschy M.A. (1993).

[47] Fodgen A. and Ninham B.W., Langmuır 7 (1991) 590.

[48] Harden J.L, Marques C., Joanny J -F and Andelman D., Langmurr 8 (1992) 1170.

[49] de Gennes P.-G., C.R. Hebd. Séan. Acad. Scı. B 275 (1972) 939.

[50] Such difficulties have been especially met with lamellar phases stabilized by undulation forces [53].

[51] Grınstein G. and Pelcovits R., Phys.Rev. Lett. 47 (1981) 856.

[52] Cowley A C., Fuller N.L., Rand R P. and Parsegıan V.A , Brochem. 17 (1978) 3163.

[53] Helfrich W., Z. Naturforsch. 33a (1978) 305 
[54] Ninham B.W. and Parsegian V.A., J. Chem Phys. 53 (1970) 3398.

[55] Asakura S. and Oosawa F , J. Chem. Phys. 22 (1954) 1255.

[56] AJdari A., Thèse, Université Paris VI (1992).

[57] Sheng P, Phys Rev. Lett 37 (1976) 1059.

[58] Sheng P., Phys Rev A 26 (1982) 1610.

[59] Als-Nielsen J., Christensen F. and Pershan P S., Phys. Rev. Lett 48 (1982) 1107.

[60] Poniewierskı A. and Sluckin T.J., Lıq. Cryst 2 (1987) 281 